\title{
Instabilities of low-latitude easterly jets in the presence of moist convection and topography and related cyclogenesis, in a simple atmospheric model
}

\section{Masoud Rostami \& Vladimir Zeitlin}

To cite this article: Masoud Rostami \& Vladimir Zeitlin (2021): Instabilities of low-latitude easterly jets in the presence of moist convection and topography and related cyclogenesis, in a simple atmospheric model, Geophysical \& Astrophysical Fluid Dynamics, DOI:

10.1080/03091929.2021.1959574

To link to this article: https://doi.org/10.1080/03091929.2021.1959574

曲 Published online: 17 Sep 2021.

Submit your article to this journal $\llbracket$

Q View related articles $₫$

View Crossmark data $\nearrow$ 


\title{
Instabilities of low-latitude easterly jets in the presence of moist convection and topography and related cyclogenesis, in a simple atmospheric model
}

\author{
MASOUD ROSTAMI ${ }^{a, b}$, VLADIMIR ZEITLIN $^{a}$ \\ ${ }^{a}$ Laboratoire de Météorologie Dynamique (LMD)/IPSL, Sorbonne Université and Ecole \\ Normale Supérieure (ENS), Paris, France \\ ${ }^{b}$ Earth System Analysis, Potsdam Institute for Climate Impact Research (PIK), Member \\ of the Leibniz Association, Potsdam, Germany
}

This is the Author's Original Manuscript (AOM); that is, the manuscript in its original form; a "preprint" . The Version of Record of this manuscript has been accepted for publication by the Journal of Geophysical \& Astrophysical Fluid Dynamics, published by Taylor \& Francis. The publisher's version is available at https://doi.org/10.1080/03091929.2021.1959574. 
Geophysical and Astrophysical Fluid Dynamics

Vol. 00, No. 00, 00 Month 20xx, 2-27

\title{
Instabilities of low-latitude easterly jets in the presence of moist convection and topography and related cyclogenesis, in a simple atmospheric model
}

\author{
MASOUD ROSTAMI $^{\dagger}$ and VLADIMIR ZEITLIN $^{\dagger}$ \\ † Laboratoire de Météorologie Dynamique/Sorbonne Université/ Ecole Normale Supérieure \\ (ENS)/CNRS, 24 Rue Lhomond, 75005 Paris, France \\ $\ddagger$ Earth System Analysis, Potsdam Institute for Climate Impact Research (PIK), Member of the \\ Leibniz Association, Potsdam, Germany \\ (Received 00 Month 20xx; final version received 00 Month 20xx)
}

A simple two-layer model, the moist-convective rotating shallow water, which allows for low-cost high-resolution numerical simulations of the dynamics of the moist atmosphere in the presence of topography, is used to identify and understand dynamical processes governing the evolution of easterly waves propagating on the background of a low-latitude easterly jet crossing a land-sea boundary, a setup crudely representing the African Easterly Jet over the West-African plateau and the Atlantic ocean. We perform a thorough linear stability analysis and identify the unstable modes of the jet, which we use then for initialisation of fully nonlinear numerical simulations. In this way we determine nonlinear evolution of unstable perturbations of the jet, both in the "dry" and moist-convective environments, and highlight essential differences between the two cases. We identify a mechanism of formation of intense lower-layer cyclonic vortices at the northern flank of the jet, and determine the influence of the land-sea contrast upon this process.

Keywords: Baroclinic instability, Moist convection, Orographic effects, African Easterly Jet, Tropical Cyclogenesis 


\section{Introduction}

Understanding tropical cyclogenesis in the North Atlantic is of obvious importance. It is well-known that the cyclogenesis is most often triggered by depressions due to the African Easterly Waves (AEW) propagating over Atlantic, (e.g. Bracken and Bosart 2000, Brammer and Thorncroft 2015, Dieng et al. 2017), and references therein. In turn, it is widely accepted that AEW are related to instabilities of the African Easterly Jet (AEJ), a low-latitude atmospheric easterly jet evolving over the West-African plateau and the Eastern Atlantic Ocean (Burpee 1972, Hsie and Cook 2005, 2008, Wu et al. 2012). Understanding the instabilities of AEJ is, thus, of primary importance, and their studies, which started almost half a century ago (Simmons 1977) still continue (Thorncroft and Hoskins 1994a, Hall et al. 2006, Diaz and Aiyyer 2015). It is known that humidity, and the contrast between the ocean and the continent play an important role in the development and intensification of AEW (e.g. Brammer and Thorncroft 2015).

In the present paper, we analyze the development and nonlinear evolution of the instabilities of low-latitude easterly jets in the presence of moist convection and the land - sea contrast with the help of a simple two-layer atmospheric model which, nevertheless, captures essential dynamical mechanisms. An advantage of the model is that inclusion of topography is simple and straightforward. The model allows for efficient low-cost high-resolution linear and nonlinear analyses of the system, and we profit from this to establish fine details of linear stability diagrams, and of nonlinear saturation of instabilities. An early precursor of this study is the work by Kuo (1978), where barotropic and baroclinic instabilities of easterly jets were analyzed, with similar motivations, in the framework of a two-layer model of the same kind, although only on the linear level and with inclusion of neither topography nor dynamical effects of moisture and condensation. The model we are working with is the socalled moist-convective rotating shallow water (mcRSW), which, in its two-layer version, was derived in Lambaerts et al. (2011b), by using vertical averaging between isobaric surfaces and Lagrangian conservation of the equivalent potential temperature in the hydrostatic primitive equations with pseudo-height as vertical coordinate. This conceptual model proved to be useful in studies of instabilities of large- scale Earth and planetary jets and vortices, and the effect of diabatic effects upon them (Lahaye and Zeitlin 2016, Rostami and Zeitlin 2017, Rostami et al. 2017, 2018). The main goal of the present paper is to show that, in spite of a highly idealized character of the model, it allows to capture the fundamental dynamical mechanisms governing the evolution of AEW. Our strategy below is similar to that of Lambaerts et al. 
(2012), where the model, for benchmarking purposes, was applied to the moist baroclinic instability of the upper-tropospheric midlatitude westerly jet on the $f$-plane. Yet the important differences, apart from the jet configuration itself, are the use of the beta-plane approximation and addition of topography with a land-sea contrast. We choose a basic state which mimics the AEJ, within the precision of the model, and perform, first, the linear stability analysis in the "dry" case, with condensation switched off, and without topography. In this way we identify the unstable normal modes of the jet, which we use then, either in a pure harmonic form, or in a form of localized wave-packets, to initialize fully nonlinear high-resolution numerical simulations, which are performed both in "dry" and moist-convective environments, and also include an idealized step-like topography mimicking the West-African plateau and the land - sea contrast. We are able in this way to highlight the role the moist convection and topography are playing in intensification of the low-level depressions created by unstable disturbances, and their eventual transformation into strong localized vortices.

The paper is organized as follows. In section 2 we present the model, introduce an appropriate scaling, and define the background jet configuration. In section 3 we give the results of the linear stability analysis of the jet configuration in the "dry" version of the model, with the condensation switched off. In section 4 we present results of numerical simulations of nonlinear evolution of the unstable disturbances in the "dry" and moist-convective environments, both without and with topography. Section 5 contains summary and discussion. We give in Appendix A the results of the stability analysis in the $f$-plane approximation, which was used for benchmarking purposes. 


\section{The model, the scaling, and the jet configuration}

\subsection{The moist-convective rotating shallow water (mcRSW) model}

We will work with the later version of the mcRSW model (Rostami and Zeitlin 2018) defined by the following set of equations:

$$
\begin{aligned}
& \partial_{t} \boldsymbol{v}_{1}+\left(\boldsymbol{v}_{1} \cdot \boldsymbol{\nabla}\right) \boldsymbol{v}_{1}+f(y) \hat{\boldsymbol{z}} \times \boldsymbol{v}_{1}=-g \boldsymbol{\nabla}\left(h_{1}+h_{2}\right)+\frac{\frac{\gamma C-\gamma^{*} V}{h_{1}} \cdot \frac{\boldsymbol{v}_{1}-\boldsymbol{v}_{2}}{2}}{\partial_{t} \boldsymbol{v}_{2}+\left(\boldsymbol{v}_{2} \cdot \boldsymbol{\nabla}\right) \boldsymbol{v}_{2}+f(y) \hat{\boldsymbol{z}} \times \boldsymbol{v}_{2}=-g \boldsymbol{\nabla}\left(h_{1}+s h_{2}\right)+\frac{\gamma-\gamma^{*} V}{h_{2}} \cdot \frac{\boldsymbol{v}_{1}-\boldsymbol{v}_{2}}{2}}, \\
& \partial_{t} h_{1}+\nabla \cdot\left(\left(h_{1}-b\right) \boldsymbol{v}_{1}\right)=\underline{-\gamma C+\gamma^{*} V}, \\
& \partial_{t} h_{2}+\boldsymbol{\nabla} \cdot\left(h_{2} \boldsymbol{v}_{2}\right)=\underline{+\gamma C-\gamma^{*} V}, \\
& \partial_{t} Q_{1}+\boldsymbol{\nabla} \cdot\left(Q_{1} \boldsymbol{v}_{1}\right)=\underline{-C+E}, \\
& \partial_{t} Q_{2}+\boldsymbol{\nabla} \cdot\left(Q_{2} \boldsymbol{v}_{2}\right)=\underline{V} . \\
& \partial_{t} W_{1}+\boldsymbol{\nabla} \cdot\left(W_{1} \boldsymbol{v}_{1}\right)=\underline{+(1-\kappa) C-P,} \\
& \partial_{t} W_{2}+\boldsymbol{\nabla} \cdot\left(W_{2} \boldsymbol{v}_{2}\right)=\underline{+\kappa C-V},
\end{aligned}
$$

where $\boldsymbol{v}_{i}=\left(u_{i}, v_{i}\right)$ is the horizontal velocity field in the layers $i=1,2$ counted from the bottom, $f(y)=f_{0}+\beta y$ is the Coriolis parameter, $\hat{z}$ is a unit vector in the vertical direction, $h_{i}$ are the thicknesses of the layers in the absence of topography, $b$ represents the topography, $g$ is the gravity acceleration, and $s=\theta_{2} / \theta_{1}>1$ is the stratification parameter, where $\theta_{i}$ are constant potential temperatures of the layers. Without the underlined terms (2.1) becomes the standard non-dissipative two-layer atmospheric shallow-water model (cf. Zeitlin 2018) with an addition of topography and two passive scalars layerwise: the specific humidity (water vapor content) integrated over the air column $Q_{i}$, and the bulk amount of liquid water $W_{i}$ in the column. Both are conserved quantities if diabatic effects are switched off. We will call the system "dry" in the latter case. The underlined terms introduce a condensation sink $C$ and a surface evaporation source $E$ in the water vapor equation in the lower layer (2.1e), and a convective flux between the layers in the mass conservation equations (2.1c), (2.1d), due to the latent heat release. This flux is proportional to the condensation, with the proportionality coefficient $\gamma$ which is defined by the underlying vertical stratification and thermodynamical parameters (cf. Lambaerts et al. 2011b). Similar parameterization is used for vaporization $V$, which is a source of water vapor, and introduces cooling with a related downward convective flux which is proportional to vaporization, with a proportionality coefficient $\gamma^{*}$. We suppose 
that the lower layer is close to saturation, and neglect vaporization in it and that the upper layer is far from the saturation and neglect condensation in it. Correspondingly, condensation (vaporization) is a source (sink) of liquid water. Entrainment of liquid water from lower to upper layer due to the upward convective flux is also introduced, and is controlled by the parameter $\kappa$. Precipitation $P$ is a sink of liquid water. The upward and downward convective fluxes lead to the Rayleigh drag terms appearing in the right-hand sides of (2.1a), (2.1b).

Relaxational parameterizations are used for the condensation $C$ and precipitation $P$, with corresponding relaxation times $\tau_{c}, \tau_{p}$ :

$$
C=\frac{Q-Q^{s}}{\tau_{c}} \mathcal{H}\left(Q-Q^{s}\right), \quad P=\frac{W-W_{c r}}{\tau_{p}} \mathcal{H}\left(W-W_{c r}\right),
$$

where $Q^{s}$ is the saturation threshold for water vapor, $W_{c r}$ is the precipitation threshold, and $\mathcal{H}$ denotes the Heaviside (step-) function. Bulk formulas are used for surface evaporation and vaporization:

$$
E=\alpha_{e} \frac{\left|\boldsymbol{v}_{1}\right|}{\left|\boldsymbol{v}_{\max }\right|}\left(Q^{s}-Q\right) \mathcal{H}\left(Q^{s}-Q\right), \quad V=\alpha_{v} \frac{\left(Q^{s}-Q\right)}{Q^{s}} W \mathcal{H}\left(Q^{s}-Q\right)
$$

where $\left|\boldsymbol{v}_{\text {max }}\right|$ is the maximum value of the lower-layer velocity, and $\alpha_{e, v}$ are adjustable coefficients. Although a threshold for evaporation can be taken to be different, we make the simplest choice and set it to be the same as in the condensation (2.2). The saturation threshold $Q^{s}$ depends on pressure according to the Clausius-Clapeyron law. This dependence, within the approximations of the model, can be taken to be linear (cf. Bouchut et al. 2009) or exponential, with a small exponent: $Q^{s}(h)=Q_{0}^{s} e^{-\epsilon \eta} \approx Q_{0}^{s}(1-\epsilon \eta)$, where $\eta$ is the thickness anomaly in the lower layer, see below. Evaporation (2.3) is the only process in the boundary layer we include in the model. Momentum dissipation due to the bottom friction can be easily included, and more involved parameterizations of the boundary layer can be used too (cf. Schecter and Dunkerton 2009), which we will not do, keeping the model as simple as possible and limiting the number of adjustable parameters. The parametrization of surface evaporation in (2.3) is well suited for the evolution of the atmosphere over the ocean (e.g. Katsaros 2001) but should be changed over land. Again, for simplicity, we simply switch it off whenever $b \neq 0$. We should emphasize that an advantage of this version of the model, as compared to the original one in Lambaerts et al. (2011b), is that it tracks condensed water, and thus is "cloud-resolving", with the distribution of $W$ giving a rough idea of the cloud cover layer-wise.

Radiative relaxation term of the form $-\left(h_{1}-H_{e}\right) / \tau_{r}$, where $\tau_{r}$ is a characteristic relaxation time, can be added in the r.h.s. of (2.1c) in the condensation regions, to model the physical process of enhanced outgoing radiation by clouds. We test the influence of this term in the 
numerical simulations below.

A detailed discussion of the conservation laws and energy budget in the model can be found in Rostami and Zeitlin (2018) and we will not repeat it here.

\subsection{Scaling of the "dry" version of the model, and the background jet configuration}

We introduce unperturbed thicknesses of the layers $H_{0 i}, i=1,2$ and the unperturbed total thickness $H_{0}=H_{01}+H_{02}$. The following scaling is used for the independent and dependent variables in the "dry" version of the model:

$$
(x, y) \sim L_{d} \equiv \sqrt{g H_{0}} / f_{0}, \quad t \sim\left(1 / f_{0}\right), \quad(u, v) \sim \sqrt{g H_{0}}, \quad(h, b) \sim H_{0},
$$

where $L_{d}$ denotes the barotropic deformation radius. For dimensional estimates we will place the beta - plane at a low latitude $\Phi_{0}$, and take $H_{0}=10 \mathrm{~km}, f_{0}=2 \Omega \sin \Phi_{0}=3.2717 \times 10^{-5} \mathrm{~s}^{-1}$ at $\Phi_{0}=13^{\circ} \mathrm{N}$, which gives $L_{d}=9568 \mathrm{~km}, \beta=2.2243 \times 10^{-11}(\mathrm{~ms})^{-1}$, and $\sqrt{g H_{0}}=313 \mathrm{~ms}^{-1}$. We recall that in the tangent-plane approximation

$$
f=2 \Omega \sin \Phi=2 \Omega \sin \Phi_{0}+\frac{2 \Omega}{R_{e}} \cos \Phi_{0} y=f_{0}+\beta y=f_{0}\left(1+\frac{\beta L_{d}}{f_{0}} y^{*}\right) \equiv f_{0}\left(1+\bar{\beta} y^{*}\right),
$$

where the asterisk denotes non-dimensional variables, and we introduced the non-dimensional beta $\bar{\beta}$. Its value is $\bar{\beta}=6.505$ at $\Phi=13^{\circ}$. The non-dimensional "dry" equations on the $\beta$-plane, after dropping the asterisks become :

$$
\begin{aligned}
& \partial_{t} u_{i}+u_{i} \partial_{x} u_{i}+v_{i} \partial_{y} u_{i}-(1+\bar{\beta} y) v_{i}=-\partial_{x}\left(h_{1}+s^{i-1} h_{2}\right), \\
& \partial_{t} v_{i}+u_{i} \partial_{x} v_{i}+v_{i} \partial_{y} v_{i}+(1+\bar{\beta} y) u_{i}=-\partial_{y}\left(h_{1}+s^{i-1} h_{2}\right), \\
& \partial_{t} h_{i}+\partial_{x}\left(\left(h_{i}-(2-i) b\right) u_{i}\right)+\partial_{y}\left(\left(h_{i}-(2-i) b\right) v_{i}\right)=0, i=1,2 .
\end{aligned}
$$

It is easy to see that in the absence of nontrivial topography, i.e. with $b=$ const, any zonal jet $u_{i}=U_{i}(y), h_{i}=H_{0 i}+H_{i}(y), v_{i}=0$ in geostrophic equilibrium:

$$
(1+\bar{\beta} y) U_{i}=-\partial_{y}\left(H_{1}+s^{i-1} H_{2}\right)
$$

is an exact stationary solution of (2.6). If $b=b(x) \neq 0$, which will be our choice below, only the upper-layer jets with quiescent lower layer $U_{1}=0$ are exact solutions. This will be the case we consider. We choose the classical Bickley jet profile for $U_{2}$, which we orient from East to West:

$$
U_{2}(y)=-U_{0} \operatorname{sech}^{2}\left[\mathrm{a}\left(\mathrm{y}-\mathrm{y}_{\mathrm{c}}\right)\right]
$$



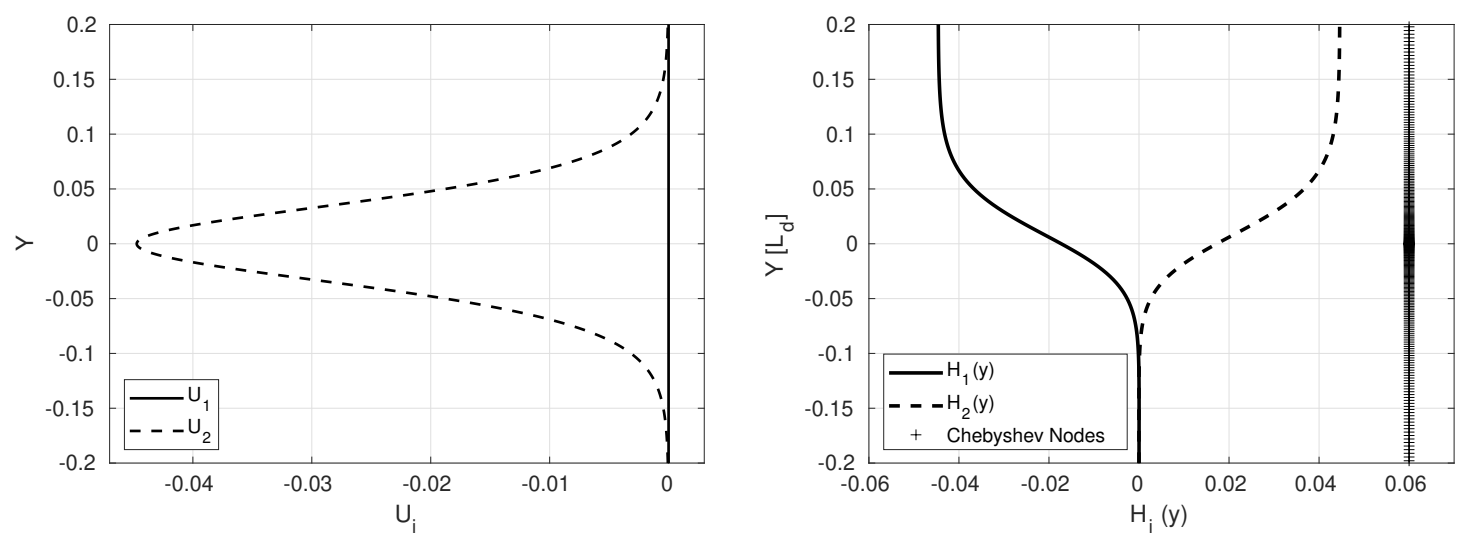

Figure 1. Meridional profiles of zonal velocities $U_{i}$ (left panel) and thickness deviations $H_{i}(y)$ (right panel). Lower (upper) layer: solid (dashed). Chebyshev nodes, which are used for spatial discretization, are shown in the right panel. $R o=0.15, B u=11.35, U_{0}=0.045, a=20, H_{01}=1 / 3, H_{02}=2 / 3, k=20, \theta_{2} / \theta_{1}=1.1, \Phi=13^{\circ} \mathrm{N}$.

where $a$ controls the width of the jet. The Burger number of the jet is defined as $B u=L_{d}^{2} / R^{2}$, where $R$ is the width of the jet at the level $0.01 U_{0}$. The position of the jet axis $y_{c}$ is variable. Instead of changing it, we choose $y_{c}=0$ and vary instead the latitude $\Phi_{0}$, and hence the value of $\bar{\beta}$ in (2.6), as it is known that this parameter is crucial for (in)stability properties (Kuo 1978). The corresponding profiles of background thickness deviations $H_{i}$ are recovered from the geostrophic balance

$$
H_{2}(y)=-\int_{-\infty}^{y} \frac{1}{s-1}(1+\bar{\beta} y) U_{2}(y) d y, \quad H_{1}(y)=-H_{2}(y) .
$$

An upper-layer jet solution, with the characteristics roughly corresponding to the AEJ (e.g. Hsie and Cook 2008) is presented in Figure 1.

\section{Linear stability analysis of the upper-layer easterly jet in the absence of topography}

We introduce small perturbations of all variables, denoted by primes, with respect to the upper-layer jet solution (2.8), (2.9):

$$
\begin{aligned}
& u_{1}=u_{1}^{\prime}, \quad v_{1}=v_{1}^{\prime}, \quad h_{1}=H_{1}(y)+\eta_{1}^{\prime}+H_{01}, \\
& u_{2}=U_{2}(y)+u_{2}^{\prime}, \quad v_{2}=v_{2}^{\prime}, \quad h_{2}=H_{2}(y)+\eta_{2}^{\prime}+H_{02},
\end{aligned}
$$

and linearize the equations (2.6) with $b \equiv 0$ about the stationary solution. Dropping the 
primes, we thus get:

$$
\begin{aligned}
& \partial_{t} u_{1}-(1+\bar{\beta} y) v_{1}+\partial_{x}\left(\eta_{1}+\eta_{2}\right)=0, \\
& \partial_{t} v_{1}+(1+\bar{\beta} y) u_{1}+\partial_{y}\left(\eta_{1}+\eta_{2}\right)=0, \\
& \partial_{t} \eta_{1}+H_{1} \partial_{x} u_{1}+v_{1} \partial_{y} H_{1}+H_{1} \partial_{y} v_{1}=0, \\
& \partial_{t} u_{2}+U_{2} \partial_{x} u_{2}+v_{2} \partial_{y} U_{2}-(1+\bar{\beta} y) v_{2}+\partial_{x}\left(\eta_{1}+s \eta_{2}\right)=0, \\
& \partial_{t} v_{2}+U_{2} \partial_{x} v_{2}+(1+\bar{\beta} y) u_{2}+\partial_{y}\left(\eta_{1}+s \eta_{2}\right)=0, \\
& \partial_{t} \eta_{2}+U_{2} \partial_{x} \eta_{2}+H_{2} \partial_{x} u_{2}+v_{2} \partial_{y} H_{2}+H_{2} \partial_{y} v_{2}=0 .
\end{aligned}
$$

We look for harmonic solutions of (3.10) in the form: $\left(u_{i}, v_{i}, \eta_{i}\right)=\operatorname{Re}\left[\left(\tilde{u}_{\mathrm{i}}, \mathrm{i} \tilde{v}_{\mathrm{i}}, \tilde{\eta}_{\mathrm{i}}\right) \mathrm{e}^{\mathrm{i}(k \mathrm{x}-\omega \mathrm{t})}\right]$ Complex eigenfrequencies $\omega=\omega_{R}+\mathrm{i} \omega_{I}$ with positive imaginary part $\left(\omega_{I}>0\right)$, correspond to instabilities with linear growth rate $\sigma=\omega_{I}$. The resulting eigenproblem is:

$$
L\left[\begin{array}{llllll}
\tilde{u_{1}} & \tilde{v_{1}} & \tilde{\eta_{1}} & \tilde{u_{2}} & \tilde{v_{2}} & \tilde{\eta_{2}}
\end{array}\right]^{\mathrm{T}}=\omega\left[\begin{array}{llllll}
\tilde{u_{1}} & \tilde{v_{1}} & \tilde{\eta_{1}} & \tilde{u_{2}} & \tilde{v_{2}} & \tilde{\eta_{2}}
\end{array}\right]^{\mathrm{T}},
$$

$L=\left[\begin{array}{cccccc}0 & -(1+\bar{\beta} y) & k & 0 & 0 & k \\ -(1+\bar{\beta} y) & 0 & -D & 0 & 0 & -D \\ k H_{1} & H_{1}^{\prime}(y)+H_{1}(y) D & 0 & 0 & 0 & 0 \\ 0 & 0 & k & k U_{2}(y) & U_{2}^{\prime}(y)-(1+\bar{\beta} y) & k s \\ 0 & 0 & -D & -(1+\bar{\beta} y) & k U_{2}(y) & -s D \\ 0 & 0 & 0 & k H_{2}(y) & H_{2}^{\prime}(y)+H_{2}(y) D & k U_{2}(y)\end{array}\right]$,

where $D$ denotes the operator of differentiation with respect to $y$, which will become the Chebyshev differentiation matrix after discretization, the superscript $\mathrm{T}$ denotes the transpose, and prime denotes the derivative of the corresponding functions with respect to their argument $y$.

We should emphasize at this point that the analysis of the spectrum of unstable modes of easterly jets on the beta-plane, as compared to their westerly counterparts, encounters difficulties related to the presence of critical levels $y=y_{c r}$ where the phase velocity of the eigenmode $c=\omega_{R} / k$ coincides with the jet velocity $U\left(y_{c r}\right)$, which appear already in the simplest case of the barotropic jet in the quasi-geostrophic approximation (e.g. Maslowe 1991, Swaters 1999). This is why a theoretical analysis of this spectrum is a challenge. Instead of 

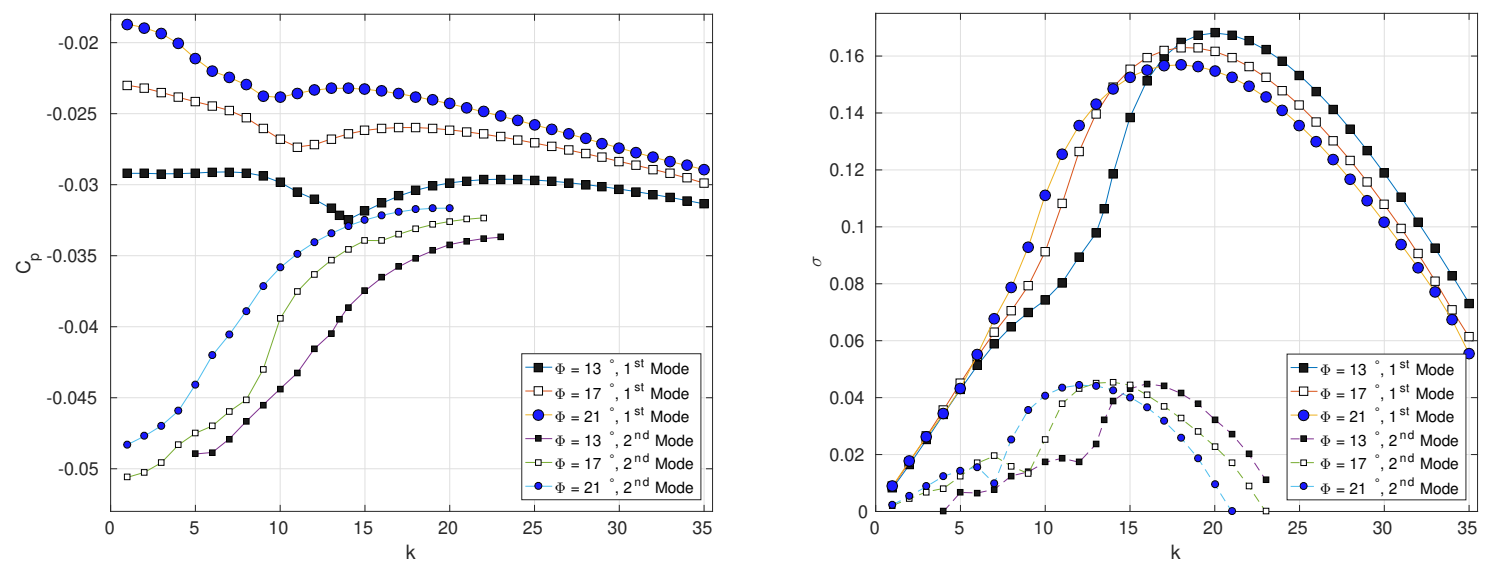

Figure 2. Stability diagrams of the upper-layer easterly Bickley jet on the $\beta$-plane at three different latitudes in terms of phase velocity $c_{p}$ (left panel) and growth rate $\sigma$ (right panel) as functions of zonal wavenumber $k$. Ro $=0.15, B u=$ $11.35, U_{0}=0.045\left[f_{0} L_{d}\right], a=20, H_{01}=1 / 3, H_{02}=2 / 3$ (Colour online).

tempting it, we adopt a pragmatic approach, consisting in a direct numerical solution of the eigenproblem (3.11), (3.12) by using pseudo-spectral collocation method with a non-uniform distribution of Chebyshev nodes which is denser inside the jet and is shown in the right panel of Figure 1. Critical levels should be treated carefully, as they can give rise to discontinuous numerical pseudo-modes. We identify them by increasing the numerical resolution, and discard. Typically, the resolution with $N=400$ collocation points in $y$ turns out to be fully sufficient. We first benchmarked the method on the $f$-plane, where the results are insensitive to the orientation of the jet on the plane, and had to reproduce those of Lambaerts et al. (2012) who studied stability of the same configuration, but in the case of a westerly jet. This is indeed the case, both for the stability diagram, and for the phase-portrait and meridional cross-section of the most unstable mode, as shown in the Appendix.

After having benchmarked the method, we perform the same analysis on the beta-plane. Stability diagram in a large range of zonal wavenumbers for three latitudinal positions of the jet, with corresponding changes of $\bar{\beta}$, is presented in Figure 2, where we display not only the first, most unstable, but also the second unstable mode. As follows from the Figure, the higher the latitude the closer, qualitatively, the results are to those on the $f$-plane, cf. Figure A1, although the growth rates on the beta-plane are higher. The curves of the phase velocity display a non-monotonicity in the interval $k \in[5,20]$, which becomes more pronounced at lower latitudes. A similar non-monotonicity was reported in Kuo (1978), although that paper used a two-layer model with a rigid lid and explored a configuration with nonzero jet velocity in the lower layer, but also in Thorncroft and Hoskins (1994a), who worked with the primitive equation model on the sphere, like Simmons (1977). We should emphasize a very good qual- 

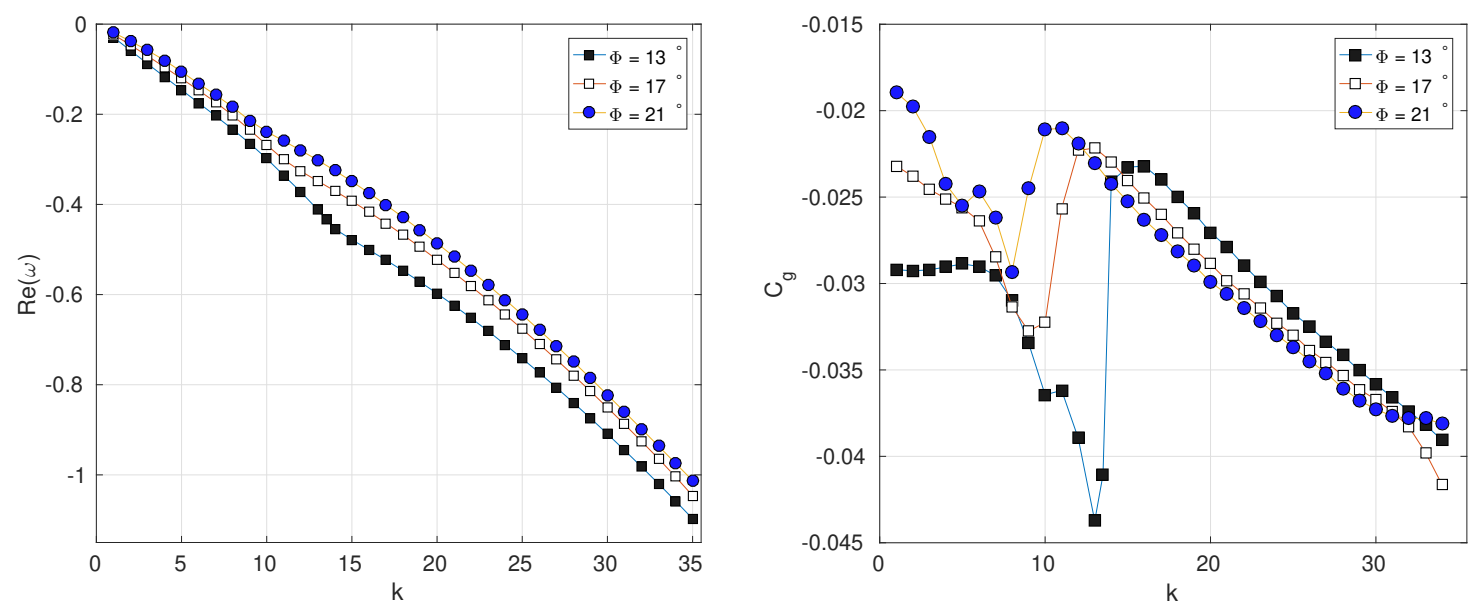

Figure 3. Real part of eigenfrequency (left panel) and group velocity (right panel) of unstable modes of Figure 2 (Colour online).

itative agreement of the stability diagram, as well as a good quantitative agreement of the zonal wavenumber, phase velocity, and growth rate of the most unstable mode in our simple model (taking into account our choice of scaling), with the results of Thorncroft and Hoskins (1994a). Note that only the most unstable mode was identified in this paper, while we find them all. What is important, the corresponding group velocity of the unstable modes, which is presented together with the real part of the eigenfrequency in Figure 3, is always westward, as the phase velocity itself, like in the case of AEW in the observations.

The phase portraits of the first and the second unstable modes of Figure 2, with the same zonal wavenumber $k=20$, are presented in Figure 4 . First, we clearly see in the Figure that the phase portrait of the most unstable mode is qualitatively similar to that of its counterpart on the $f$-plane, cf. Figure A2, although it is subject to deformations due to the beta-effect, especially in the lower layer. Second, we see that the first unstable mode is varicose, i. e. having anomalies of the same sign at both sides of the jet at a given longitude, and the second unstable mode is sinuous in the upper layer, where the jet is located, a general fact which is well-known for jet instabilities. Note that baroclinic character of the unstable mode is more pronounced at the southern side of the jet. The instability in question can be, as usual, interpreted as a resonance between Rossby waves counter-propagating across the meridional gradients of potential vorticity (cf. Thorncroft and Hoskins 1994a). An important question raised in the literature (Hall et al. 2006) is sensitivity of the results of the stability analysis to the parameters of the jet. Within our simple model, and with our simplest parameterization of the jet, a study of sensitivity is straightforward. The sensitivity of the results to the latitudinal position of the jet is clear from Figure 2: the lower the latitude of the jet center, the larger the 

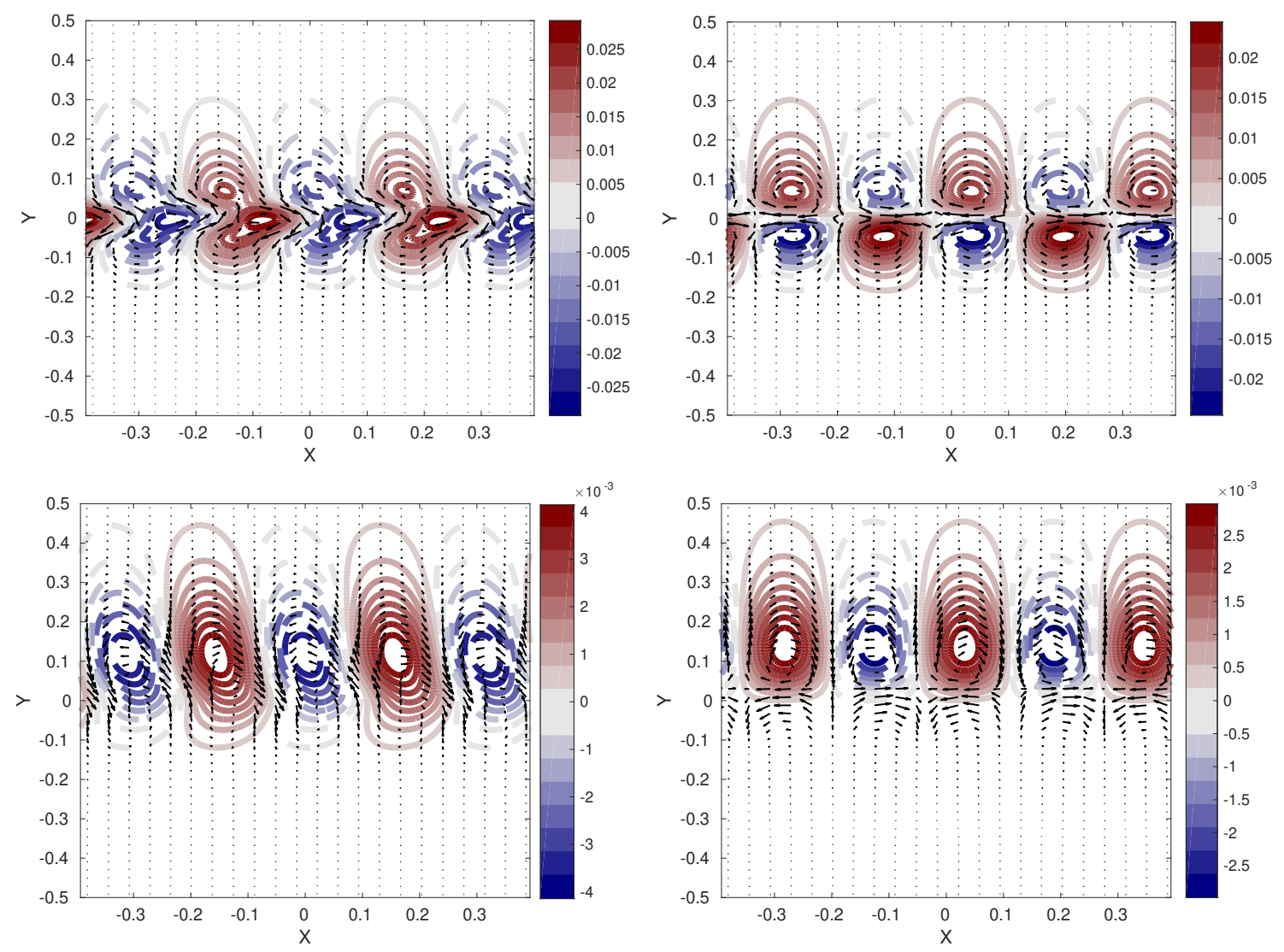

Figure 4. Geostrophic stream-functions of the unstable modes on the $\beta$-plane in the upper layer (upper row) $\left[\psi_{2}=\right.$ $\left.\eta_{1}+s \eta_{2}\right]$ and the lower layer (lower row) $\left[\psi_{1}=\eta_{1}+\eta_{2}\right]$ of the first (left column) and the second (right column) unstable modes. Positive (negative) values are represented by solid (dashed) lines, and the velocity field is represented by arrows. $R o=0.15, U_{0}=0.045, B u=11.35, a=20, H_{01}=1 / 3, H_{02}=2 / 3, k=20, \Phi=13^{\circ} \mathrm{N}$ (Colour online).

wavenumber of the most unstable mode. The sensitivity to the value of the Burger number, i.e. to the width of the jet, can be inferred already from Figure A1. The sensitivity to the Burger number on the $\beta$ - plane is presented in Figure 5 . As follows from these figures, at lower $B u$, i.e. for wider jets, the wavenumber of the most unstable mode diminishes, and at higher latitudes the wavenumber of the most unstable mode shifts down, as well.

The results of the analysis of sensitivity to the aspect ratio and stratification are presented, respectively, in Figures 6 and 7, which show that these sensitivities are rather low in the interval of wavenumbers corresponding to the highest (and, thus, most interesting) growth rates.

We should finally comment on the role of the bottom friction, which is easy to include in the model, as already mentioned. We, however do not do this, as the lower layer in our basic state is quiescent, and the amplitude of velocity of the unstable modes in this layer is an order of magnitude lower than in the upper layer, cf. Figure 4. Yet in the configuration with lower-layer westerlies, which we neglect, the lower layer damping does influence the structure 

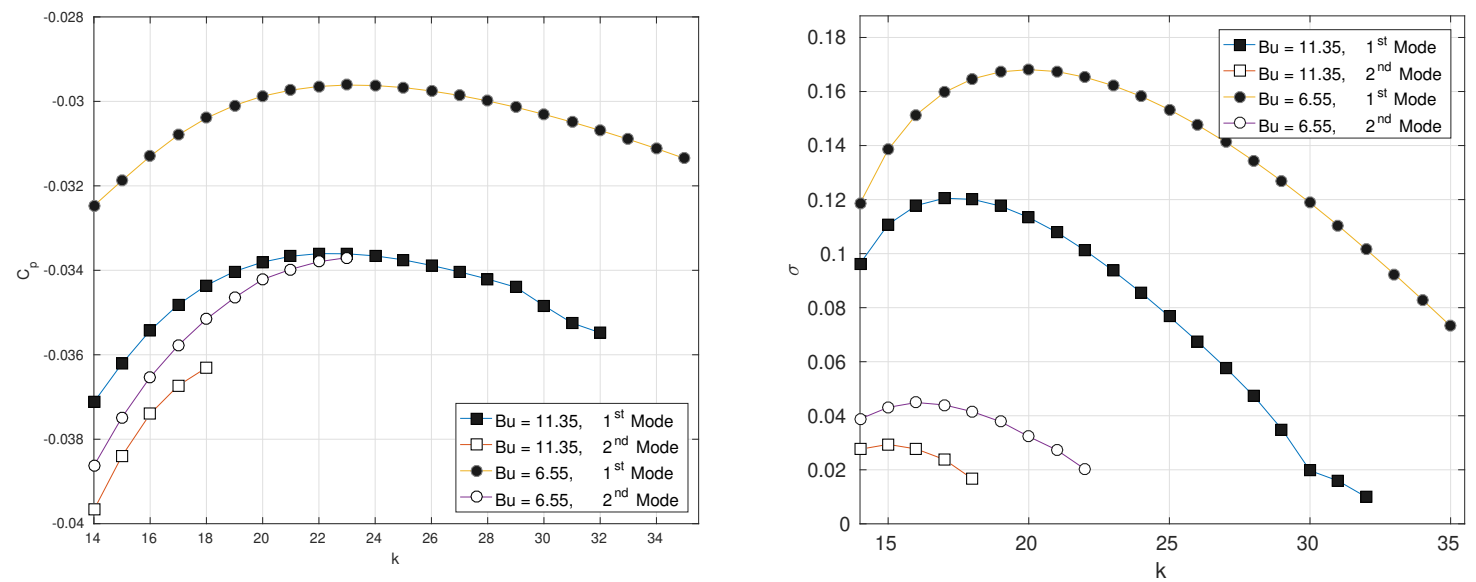

Figure 5. Sensitivity of the stability diagram, in the region of the highest growth rates to the value of $B u$. Phase velocity $c_{p}$ (left panel) and growth rate $\sigma$ (right panel). $R o=0.15, U_{0}=0.0447\left[f_{0} L_{d}\right], k=20, s=1.1, H_{01}=1 / 3, H_{02}=2 / 3$.
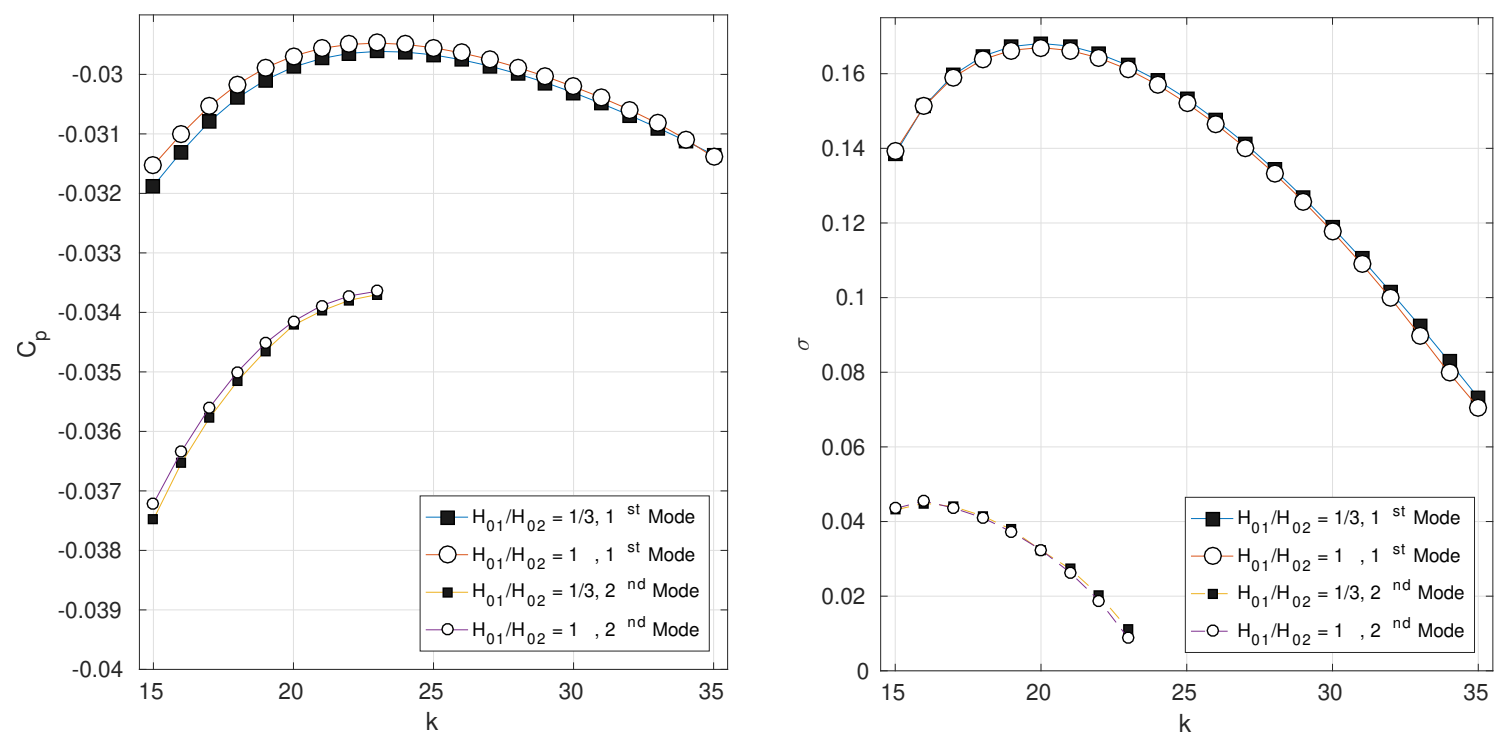

Figure 6. Sensitivity to the ratio of the layers' thicknesses of the stability diagram for phase velocity $c_{p}$ (left panel) and growth rate $\sigma$ (right panel). $R o=0.15, B u=11.35, U_{0}=0.045\left[f_{0} L_{d}\right], a=20, k=20, s=1.1, H_{01}+H_{02}=1$.

of the normal modes (Hall et al. 2006).

\section{Nonlinear evolution of the unstable disturbances}

\subsection{Parameters and setup}

To investigate the nonlinear evolution of the instabilities of the jet, we used a high-resolution finite-volume code by Bouchut and Zeitlin (2010). The simulations were set in the domain with the zonal dimension $L_{x}=n \lambda, n=5$, in units of $L_{d}$, where $\lambda$ is the wavelength of an 

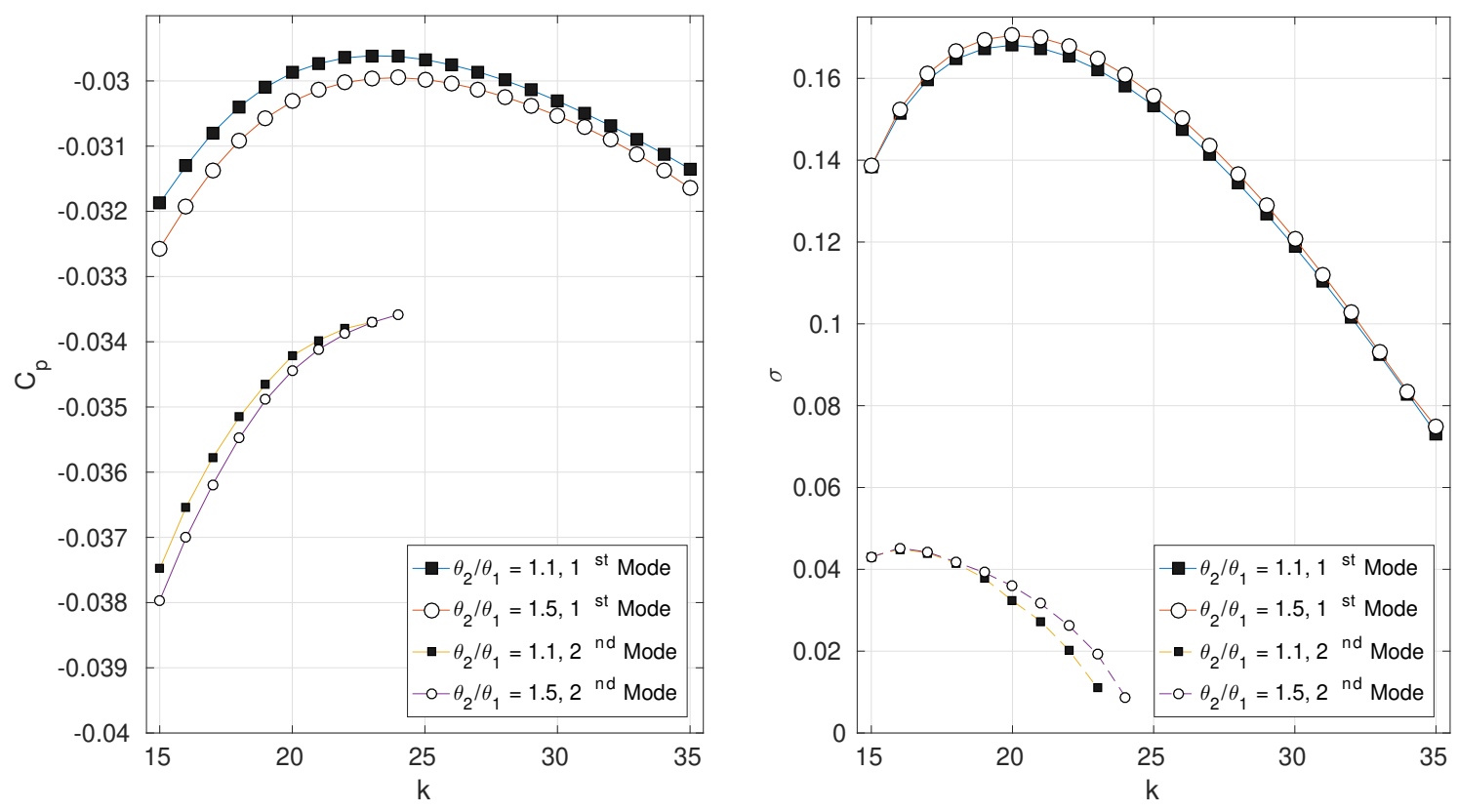

Figure 7. Sensitivity to stratification of the stability diagram for phase velocity $c_{p}$ (left panel) and growth rate $\sigma$ (right panel). $R o=0.15, B u=11.35, U_{0}=0.045\left[f_{0} L_{d}\right], a=20, k=20, s=1.1, H_{01}=1 / 3, H_{02}=2 / 3$.

unstable mode used in the simulation, and meridional dimension $L_{y}=3.8 L_{d}$. A "continent" in the simulations with topography was represented by a meridional band of width $0.7 L_{d}$, of uniform height $b=0.05 H_{0}$. (The results presented below do not qualitatively vary with changes of this value, within the same order of magnitude). Periodic and sponge boundary conditions were implemented, respectively, in zonal (along-jet), and meridional (cross-flow) directions. Notice that the periodic boundary conditions imply that the background jet has uniform intensity across the domain, which is, obviously, an idealization of the observed AEJ. We should also say that, concerning the land-sea boundary, the model, by construction, cannot capture the associated thermal contrast. To do this, a variant of the model, which was recently proposed in Liu et al. (2020), and allows for horizontal temperature gradients, is necessary. This development is postponed to a future work.

The following values of parameters characterizing the moist processes were used in the simulations: $\gamma=0.3, \gamma^{*}=0.1 \gamma, \alpha_{e}=0.25, \epsilon=0.3, Q^{s}=0.9, W_{c r}=0.01$. The simulations in the moist-convective environment were initialized with a uniform, humidity in the lower layer, close to saturation, according to the hypotheses of the model: $Q_{1}=Q^{s}-0.01$ over the ocean, and far from the saturation $0.2 Q^{s}$ over the land. The humidity in the upper layer was taken to be far from the saturation $\left(Q_{2}=Q^{s}-0.2\right)$ The liquid water content was set to be zero everywhere at the initial moment. The difference between the evaporation properties 
between the land and the sea was accounted for in the simplest way: the evaporation was switched off over topography. The condensation and precipitation relaxation times were taken to be $\tau_{c}=120 \delta_{t} \approx 2 h, \tau_{p}=30 \delta_{t}$, respectively, where $\delta t=0.002$ is the non-dimensional time-step of the code. Increasing the relaxation time of condensation within the same order of magnitude does not change the results qualitatively. The non-dimensional vaporization coefficient was chosen as $\alpha_{v}=\left(10 \cdot Q^{s} \tau_{c}\right)^{-1}$ We also compared simulations without and with radiative cooling, with the relaxation time 40 days in the latter case.

\section{2. "Dry" vs moist-convective simulations without topography initialized with the most unstable mode}

We start with the analysis of nonlinear evolution of the most unstable mode in the absence of topography in the "dry" configuration, by initializing the simulations with the background jet with superimposed small $(\approx$ one tenth of percent, compared to the values in the jet itself) perturbation of all fields of an unstable mode. The corresponding evolution of relative vorticity is presented in Figure 8, and reveals no characteristic comma-shaped pattern of a breaking unstable wave, which is typical for midlatitude westerly jets (Lambaerts et al. 2011a). Nevertheless, a steepening of the streamlines, which was called "breaking AEW" in the analysis of observations (Cornforth et al. 2017, Lafore et al. 2017), does take place. After a period of initial growth a nonlinear saturation at finite amplitude takes place. At the same time, lateral spreading of vorticity anomalies in the lower layer is observed. What is important in the following, this spreading is meridionally asymmetric, extending farther at the northern than at the southern flank of the jet. As is easy to check by using the values of the phase velocity and frequency from Figures 2, 3, neither of them matches their counterparts for free Rossby waves estimated from the quasi-geostrophic expression $\omega=-\bar{\beta} k /\left(k^{2}+l^{2}+L_{d}^{-2}\right)$, where $l$ is meridional wavenumber of the free wave, and $L_{d}$ is deformation radius (barotropic or baroclinic). Analysis of the evolution of divergence (not shown) reveals no significant emission of inertia-gravity waves either. Hence, the origin of the saturation of the instability is not a loss of energy by radiation, and is essentially nonlinear. It is not a breaking of the unstable mode, like in westerly mid-latitude jets, either, but a repartition of energy through the spectrum of unstable modes. This is confirmed by the Fourier analysis of the dynamical variables, which reveals the appearance and growth of modes with lower values of $k$ during the process of saturation, not shown. (Notice that subharmonic modes were excluded in Lambaerts et al. (2012) by the choice of boundary conditions, unlike the present case).

As compared to the "dry" simulation of Figure 8, the evolution of the instability in the moist- 

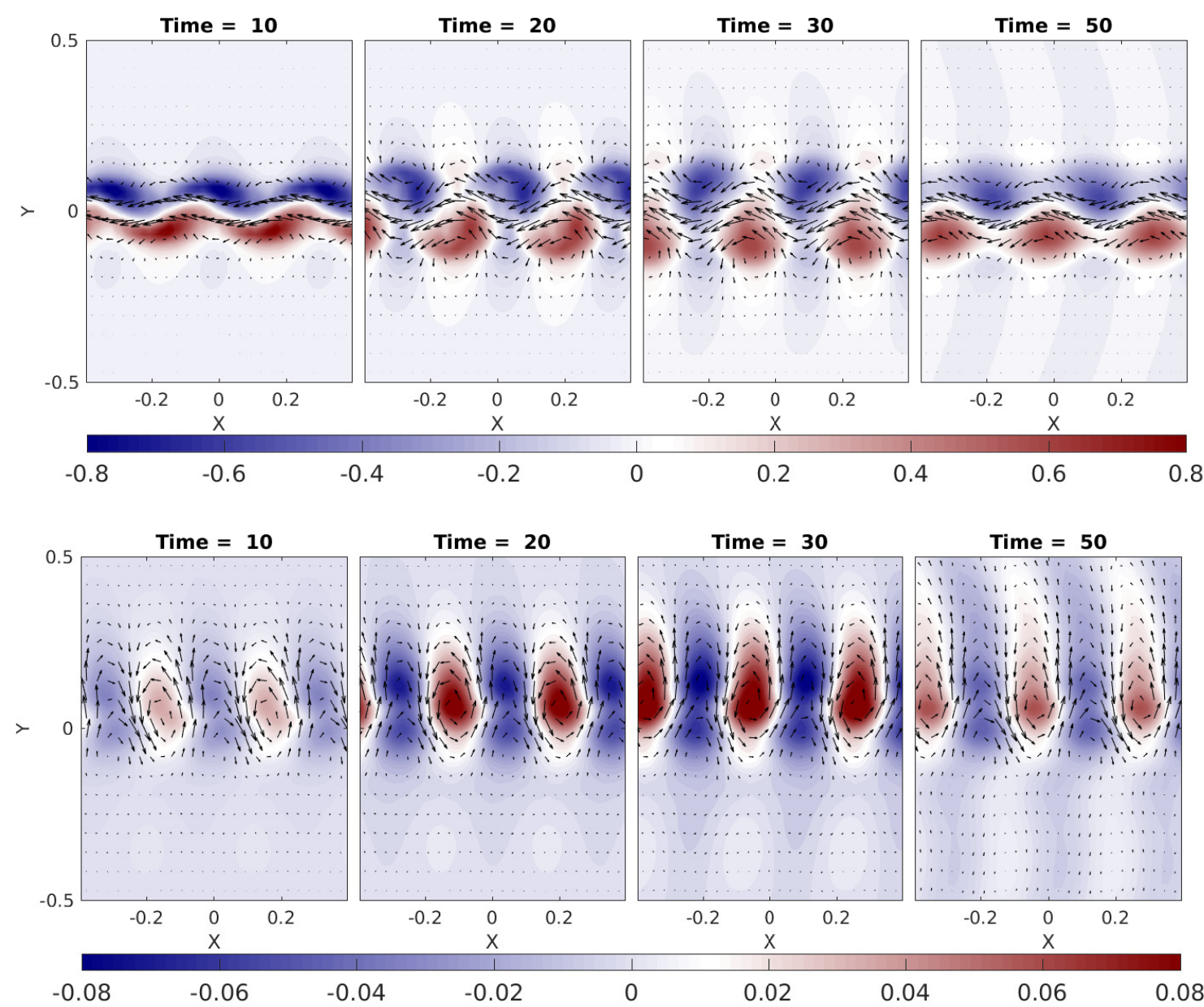

Figure 8. Evolution of vorticity and velocity in the upper (upper row) and lower (lower row) layer s in "dry" environment in a simulation initialized with the full periodic unstable mode of small non-dimensional amplitude (0.005), without radiative cooling. The global configuration is the same as in Figure 4 . The length unit at the axes here and below is $L_{d}$ (Colour online).

convective (MC) environment is totally different, as follows from Figure 9. The meridionally asymmetric periodic zones of cyclonic vorticity developing from their counterparts in the initial perturbation, like in the dry case, undergo a different evolution under the influence of the moist convection. At earlier stages they are intensified due to the moist convection. The mechanism of this intensification in the present model is very simple, and consists in growth of cyclonic vorticity due to the convective mass sink, as explained in Lambaerts et al. (2011a). As follows from Figure 9, the condensation is correlated with the enhanced convergence zone inside of the vorticity anomaly, which is shifted north-east. Convergence is a proxy for ascent in shallow-water models, so what is observed in the Figure is consistent with the discussion of the position of maximum ascent in Thorncroft and Hoskins (1994a,b). At later stages a secondary lower-layer jet, which is retrograde with respect to the primary one, is formed close to the axis of the original upper-layer jet. Interestingly, retrograde jets in the lower 
troposphere are typically accompanying the AEJ in the observations (Cook 1999), so here we see a dynamical mechanism of their generation. As follows from the Figure, this jet is formed by merging cyclonic anomalies of relative vorticity, which are induced by the unstable mode, are growing due to the instability, and are enhanced by the condensation. On the other hand, localized zones of enhanced cyclonic vorticity are formed at the northern flank of the jet. No such cyclogenesis is observed at the southern flank, due to meridional asymmetry of the unstable mode. As follows from Figure 10, where we present the late stages of the simulation of Figure 9, the cyclones that form at the northern flank of the jet are further intensified due to the upward convective fluxes, as explained in Lambaerts et al. (2011a), and have a tendency to detach from the jet. Transformation of positive vorticity into detached cyclones is not fast and takes few weeks to occur. This suggests an importance of non-modal growth, and corresponding initializations of non-linear simulations, see below. Such properties of cyclogenesis by AEJ, and asymmetry between the northern and southern flanks of the AEJ, have been reported in data analyses (e.g. Pytharoulis and Thorncroft 1999, Chen et al. 2008). The cloud cover presented in the bottom-right panel of the Figure is in qualitative agreement with observations, in a sense that condensation is within and eastward of the trough, but more complicated cloud structures were also detected in the observations, e.g. Cornforth et al. (2017) and references therein.

The left and the right panels of Figure 11 display Hovmöller diagrams of vorticity corresponding to the above-described "dry" and moist-convective experiments, and clearly show the westward propagation of disturbances, and their intensification in the moist-convective case.

We also repeated the same simulation with radiative relaxation. Qualitatively, the scenario of the evolution does not change, but the intensity of the vortex and jet structures generated by the developing instability decreases at later stages, not shown.

\subsection{Evolution of localized wave-packets of unstable modes, and the role of the land-ocean contrast and topography}

In order to render our simulations more realistic, we also initialized them with a localized wave-packets of the unstable modes. An example of such initial condition is given in Figure 12. It is obtained by taking a Gaussian envelope in the zonal direction of all fields corresponding to the most unstable mode, with a width roughly corresponding to two wavelengths of the latter. (It is possible, by solving a "dry" adjoint problem, to identify the most rapidly growing non-modal perturbation, following e.g. Parker (2008) in the present context, but we 

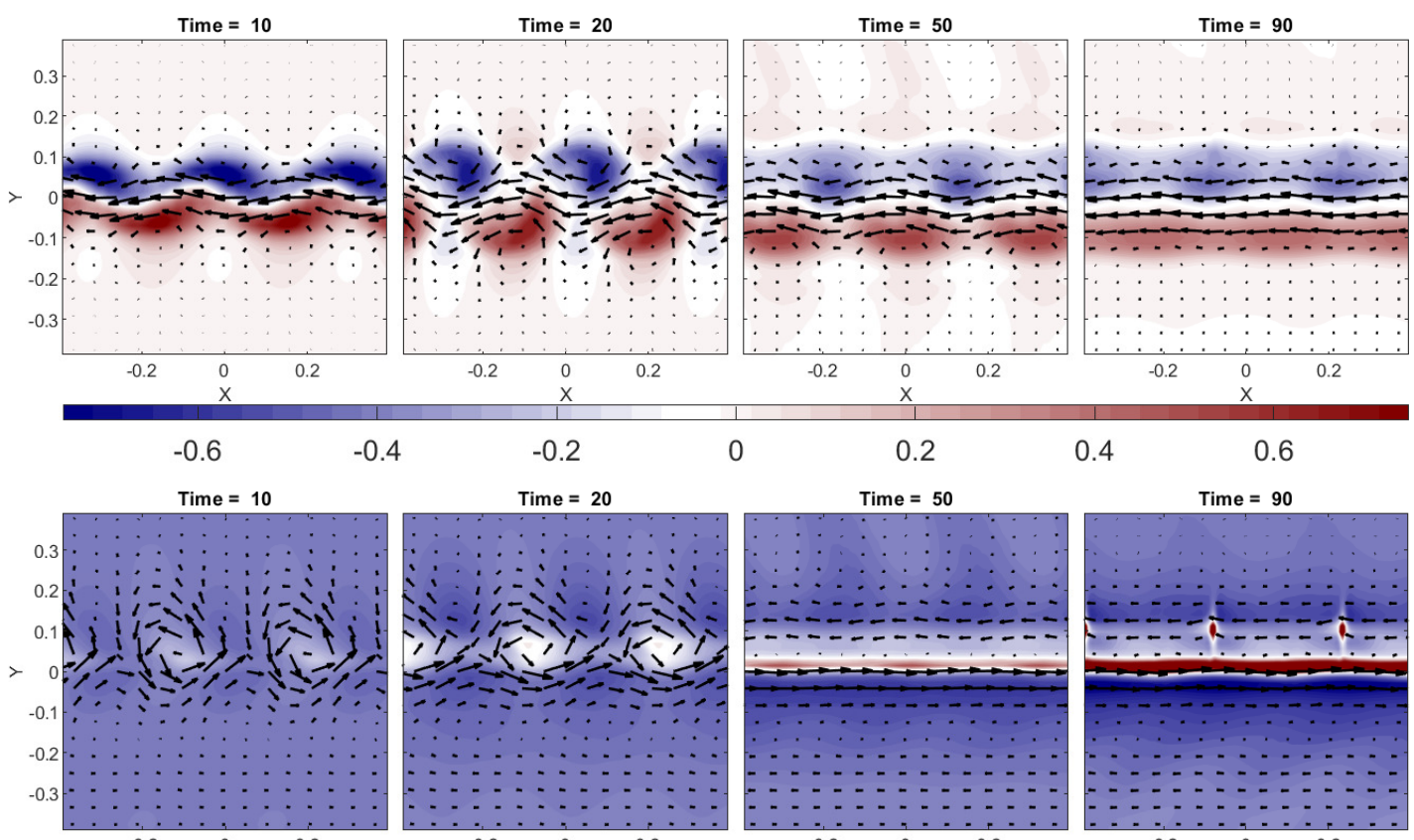

0.4

0.6

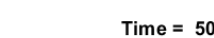

Time $=90$

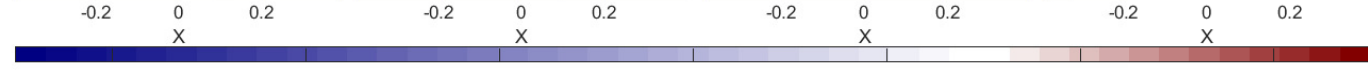

$-0.2$

$-0.1$
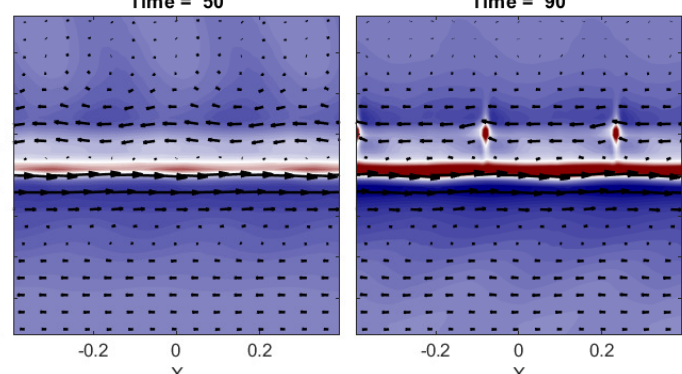

0.1

0.2

0.3

0.4

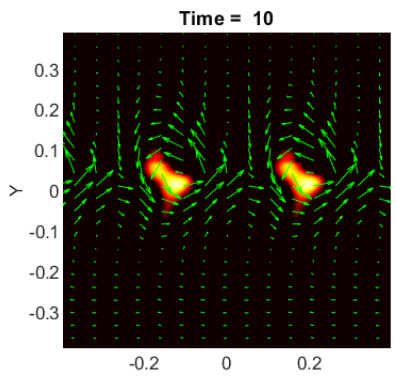

Time $=20$
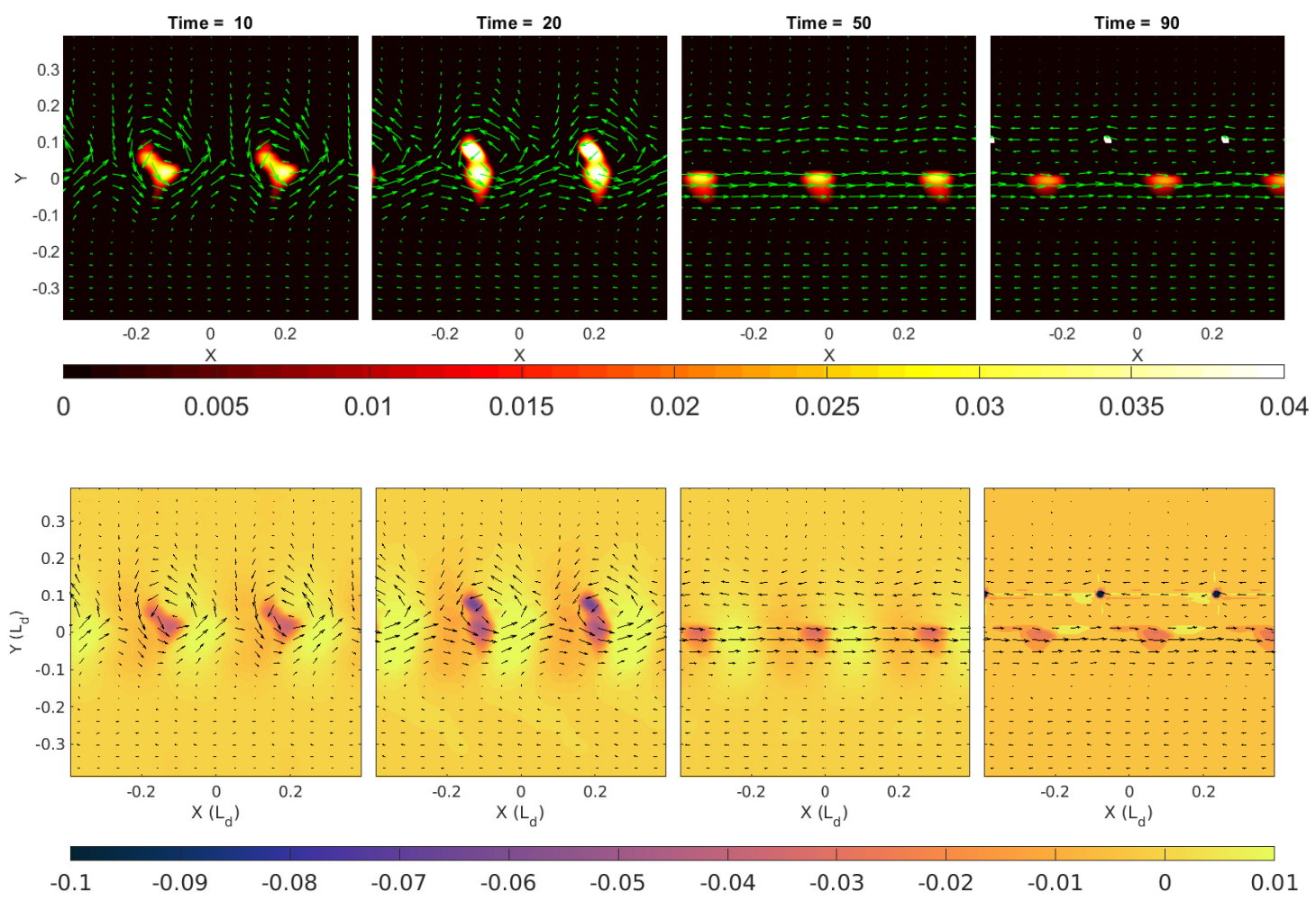

Figure 9. Evolution of the most unstable mode in moist-convective environment, as seen in the vorticity and velocity fields in the upper (upper row) and lower (second row) layer. Corresponding evolution of condensation (third row) and divergence (fourth row) in the lower layer. Same simulation as in Figure 8, but with condensation and evaporation switched on (Colour online). 

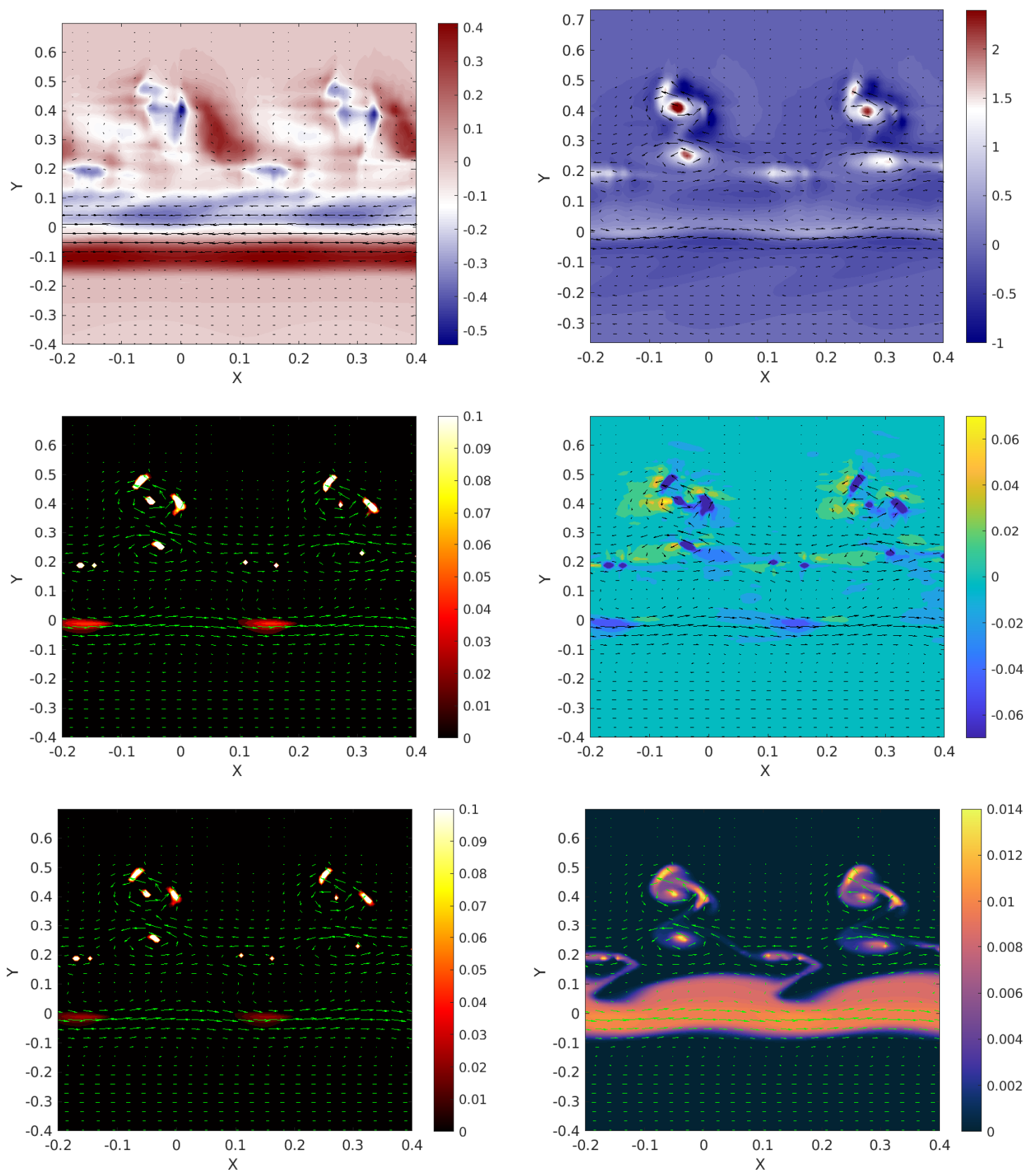

Figure 10. First row: Late stages of the evolution of vorticity and velocity in the upper (left panel) and lower (right panel) layers illustrating cyclogenesis in the lower layer due to the moist-convection. Second row: Corresponding condensation (left panel) and divergence (right panel) fields in the lower layer. Third row: Corresponding precipitation (left panel) and clouds (right panel). To distinguish various details, we made a zoom on two adjacent detaching vortices (Colour online).

adopt here a more straightforward approach). We analyze the evolution of the jet with superimposed perturbation of Figure 12 in "dry" (not shown) and moist-convective environments, and determine the role in this process of topography and the land-ocean boundary, which are roughly mimicking the West-African plateau, The height of the plateau is taken to be constant $b=0.05 H_{0}$, and have zonal extension $[-0.350 .35]$ in non-dimensional terms. The nonlinear 

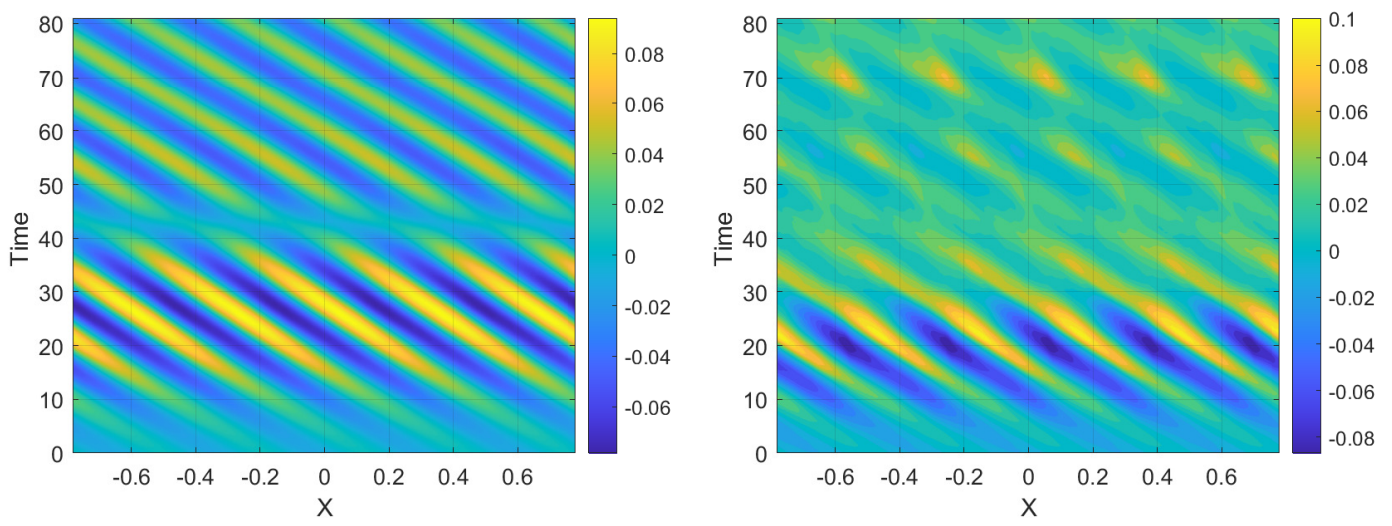

Figure 11. Hovmöller diagrams of vorticity in the lower layer at $y=0.1$ in "dry" (left panel) and moist-convective (right panel) environments corresponding to Figs. 8 and 9, respectively. Note that colorbars are not the same in the panels, for better visibility (Colour online).
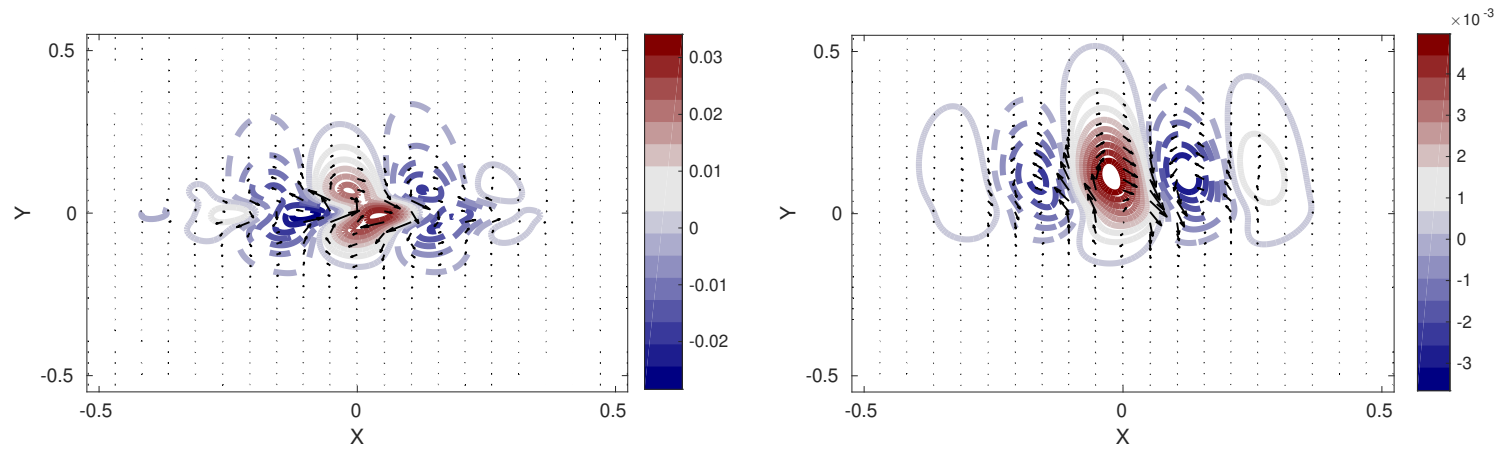

Figure 12. Initial condition as a wave packet constructed with the most unstable mode. Left (right) panel: vorticity and velocity in the upper (lower) layer. Global configuration is the same as in Figure 4. The maximal non-dimensional amplitude of the perturbation is 0.005 (Colour online).

simulations are initialized with wave-packets of unstable modes over the land, as this is the case in the observations of AEW. The evolution of the wave-packet in the moist-convective environment in such configuration is presented in Figure 14. The corresponding development of clouds and precipitation is shown in Figure 15. As follows from the Figures, the wave-packet, as expected, is subject to dispersion, accompanied by wave emission. At the same time, weak lower-layer vorticity anomalies induced by the initial condition are growing in intensity, due to the instability of the carrier mode, cf. Figure 9. There is practically no difference between the evolution with and without topographic elevation in the dry case, except for a weak topographic Kelvin wave propagating along the eastern coast (the corresponding signal, which is enhanced in the presence of moist convection, can be distinguished in the right-bottom panel of Figure 14), as the "continental plateau" is low - as already explained, we consider a configuration which is similar to the real one, within the precision of the model. However, the land-sea contrast in the moist-convective case is important. Cyclonic vorticity anomalies due to initial perturbation move over the "continent" practically without condensation, but 

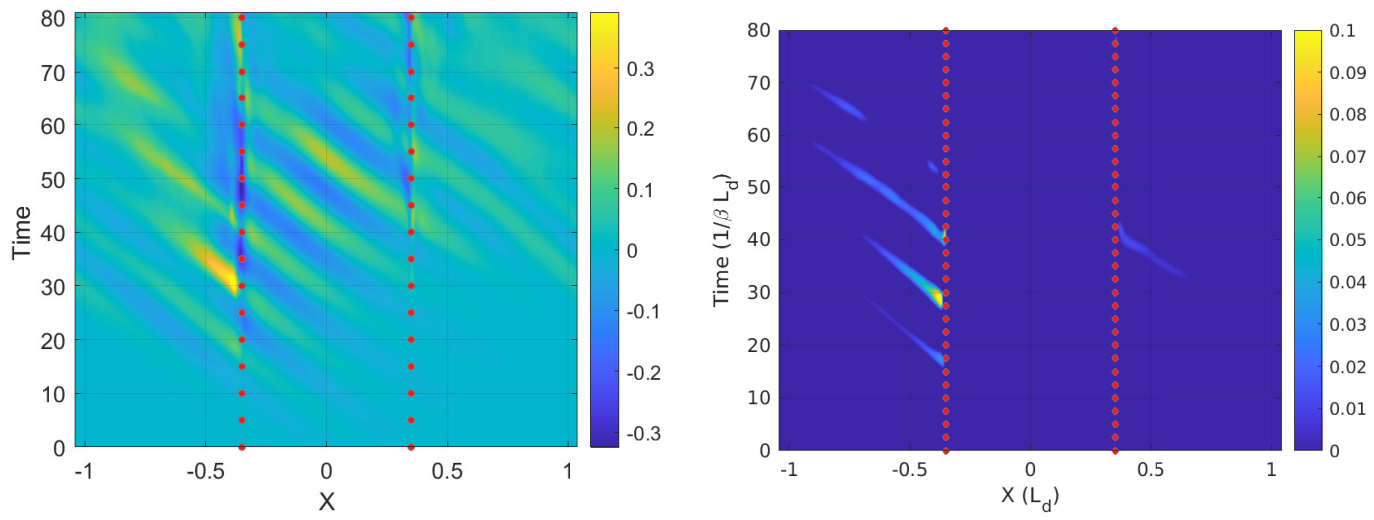

Figure 13. Hovmöller diagrams of vorticity (left panel) and condensation (right panel) in the lower layer corresponding to vorticity anomalies crossing the land-sea boundary (Colour online).

when they cross over the ocean the condensation due to evaporation switches on and leads to their rapid intensification. The result of this process is an appearance of a series of intense vortices, detaching from the continent and moving westward. The Hovmöller diagrams of vorticity and condensation presented in Figure 13 illustrate this process. Due to periodicity in zonal direction, these intense vortices eventually hit the continent at its eastern side. In the idealized setup of the present experiments, with the easterly jet of uniform intensity extending through the whole zonal extension of the domain, the encounter with the eastern continental boundary happens before the vortex has time to clearly detach from the jet, as in Figure 10. In real life, the intensity of the jet decreases across the Atlantic, and the detached vortices propagate North-West on the $\beta$-plane. The evolution of the hurricane-like vortices of different intensities was recently studied within the mcRSW model (Rostami and Zeitlin 2020), so we refer to this paper for the details of the behavior of the detached cyclones at later stages.

We should emphasize that, in the context of AEJ, the value of the group velocity of the perturbation is important, as it defines the speed of propagation of the wave-packet. We repeated the simulations of Figure 14 with a wave-packet built with the mode $k=13$ with the fastest of all unstable modes group velocity, cf. Figure 3. The results of this, and other experiments, where we combined different unstable modes to construct initial wavepackets are qualitative and quantitatively similar (not shown).

\section{Summary and Discussion}

We investigated development of instabilities of an easterly jet configuration in the presence of moist convection and land-sea contrast in a simple atmospheric model, which allows for lowcost high-resolution linear and nonlinear numerical analyses. We established linear stability 

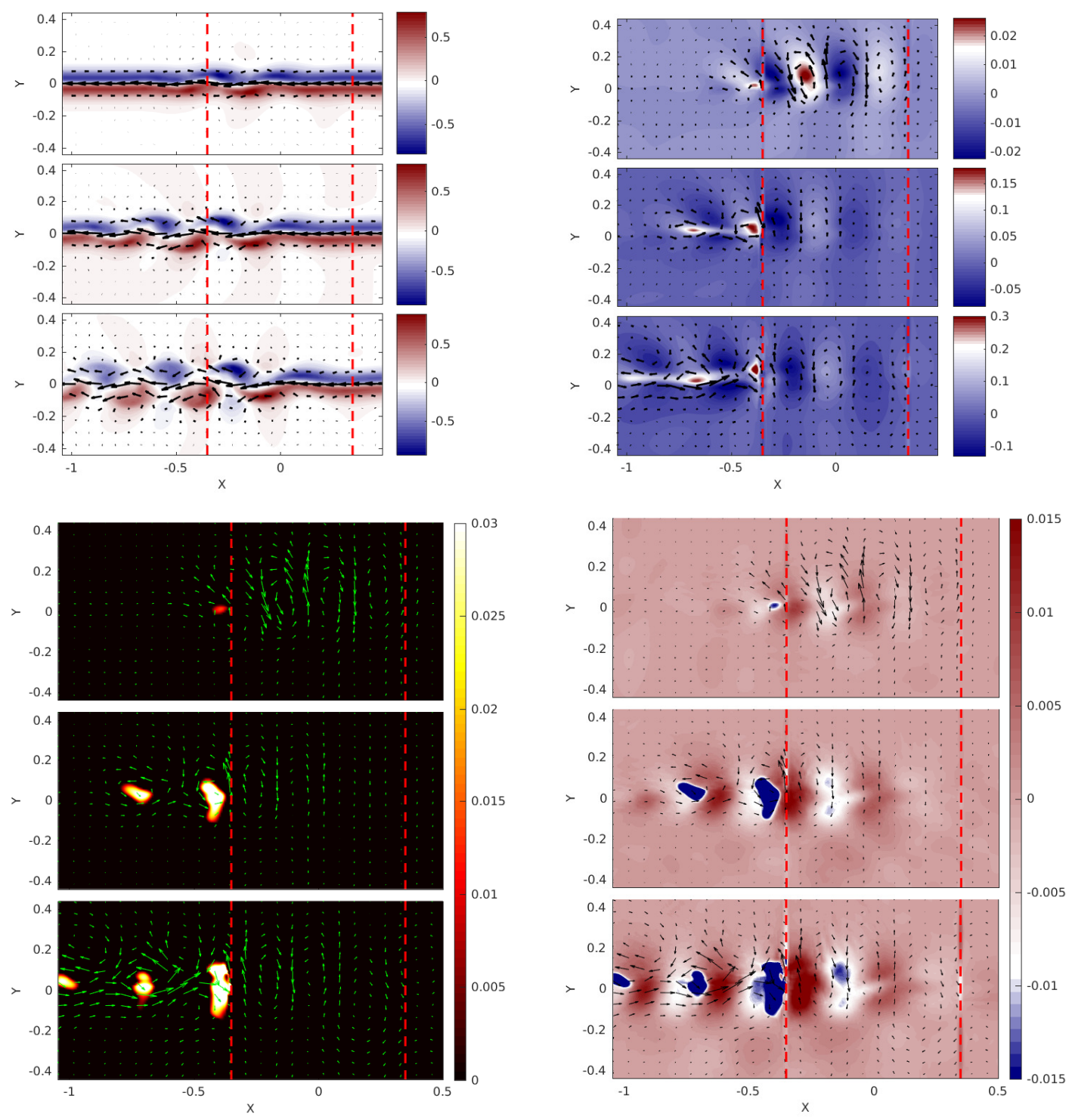

Figure 14. Upper row: Evolution of vorticity and velocity in the presence of "continental plateau" of non-dimensional height $b=0.05$, and of moist convection in the upper layer (left panel) and lower layer (right panel). Lower row: The corresponding evolution of condensation (left panel) and divergence fields (right panel). Simulations were initialized with the wave packet of Figure 12, no radiative relaxation. Notice a difference in the colorbar variation of vorticity field. Vertical dashed lines represent the borders of the "continent". Time=10, 20, 30 from up to down (Colour online).

diagrams for different parameters of the jet, and identified the unstable modes. We showed that the mechanisms of nonlinear saturation of the instability of the jet with respect to the perturbation in a form of the most unstable mode are significantly different in adiabatic and moist-convective environments. We demonstrated that saturation of the instability in the "dry" case is due to subharmonic interactions and, thus is sensitive to boundary conditions. In the moist-convective case we demonstrated a robust generation of a secondary westerly jet and of localized zones of intense cyclonic vorticity in the lower layer at the northern flank of the jet, similarly to observations. It is the structure of the most unstable mode of the jet in the lower 

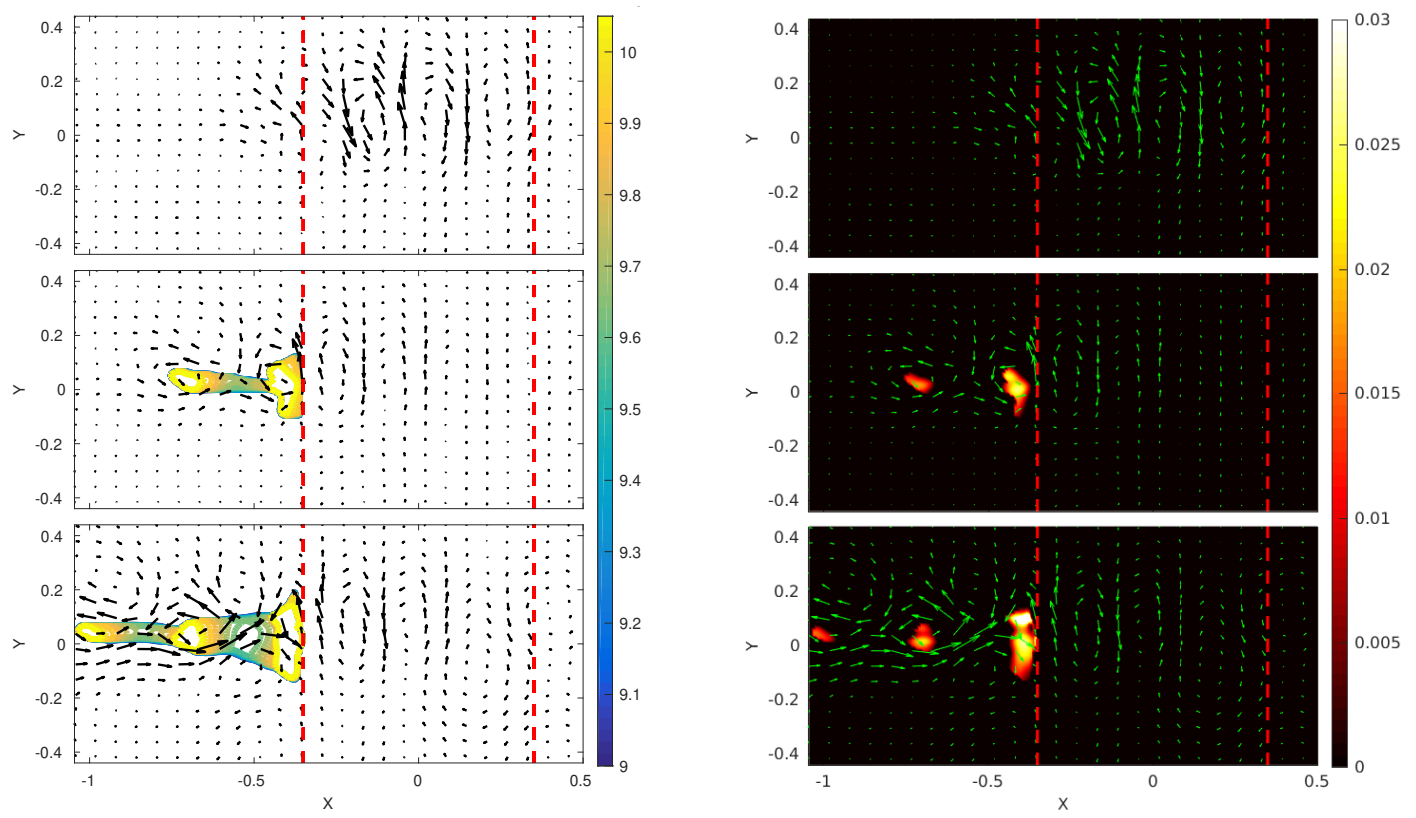

Figure 15. Evolution of high levels of precipitable water in the lower layer (left panel), with "continental plateau" of non-dimensional height $\mathrm{b}=0.05$ delimited by dashed lines, and the corresponding evolution of precipitation (right panel) (Colour online).

layer, with its meridional dissymmetry, combined with the effect of enhancement of cyclonic vorticity by moist convection which are at the origin of the cyclogenesis process. In a more realistic case of initialization of numerical experiments with localized wave-packets of unstable modes over topography mimicking a West-African plateau, we demonstrated that intense localized vortices are formed in the moist-convective environment over the ocean, to the west of the "continent" and move westward. The low flat topography does not significantly influence the scenarios of evolution of the perturbations of the jet, except for a weak topographic Kelvin-wave signal. On the contrary, the changes of humidity and evaporation at the land-sea boundary are important for enhancement of the perturbations.

Thus, in spite of a highly idealized setup the simple two-layer moist-convective rotating shallow water model does capture essential features of the development of African Easterly Waves, and allows to get insights on the fundamental dynamical mechanisms underlying the observed structures.

Acknowledgements This work was supported by the French National Program LEFEMANU. We are grateful to Doug Parker for helpful suggestions and references.

Declaration of Interests The authors report no conflict of interest. 

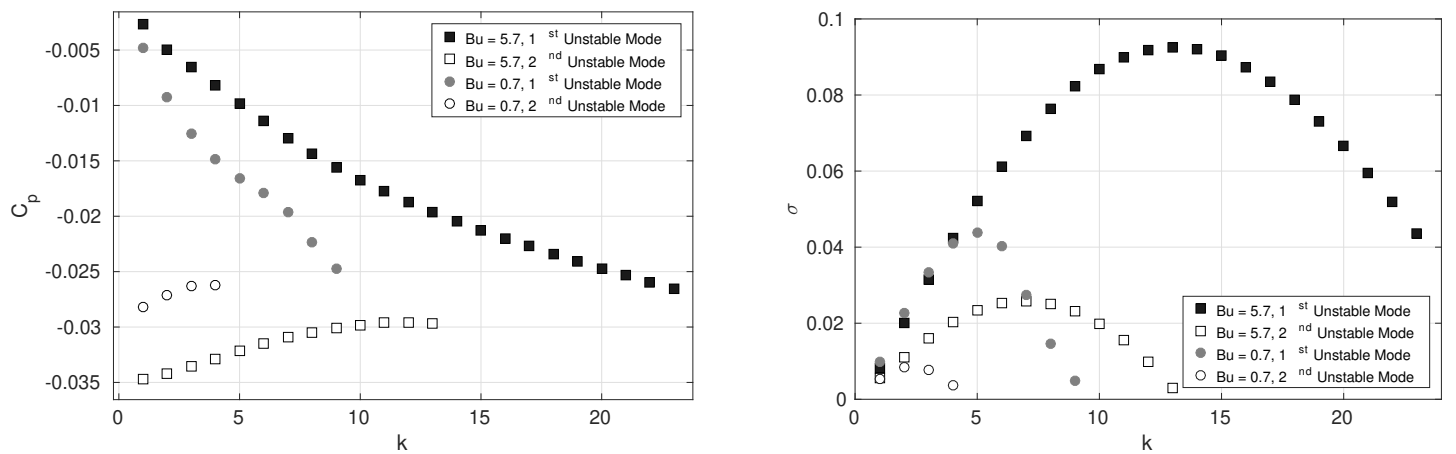

Figure A1. Stability diagram of the upper-layer easterly Bickley jet on the $f$-plane with $B u=5.7$ and $B u=0.7$ in terms of phase velocity $c_{p}$ (left panel) and growth rate $\sigma$ (right panel) as functions of the zonal wavenumber $k$. The first and the second unstable modes are represented by black and white squares for $B u=5.7$ and by gray and white circles for $B u=0.7$, respectively. $R o=0.0467, U_{0}=0.03, a=12, H_{01}=H_{02}=0.5, \bar{\beta}=0.5$.
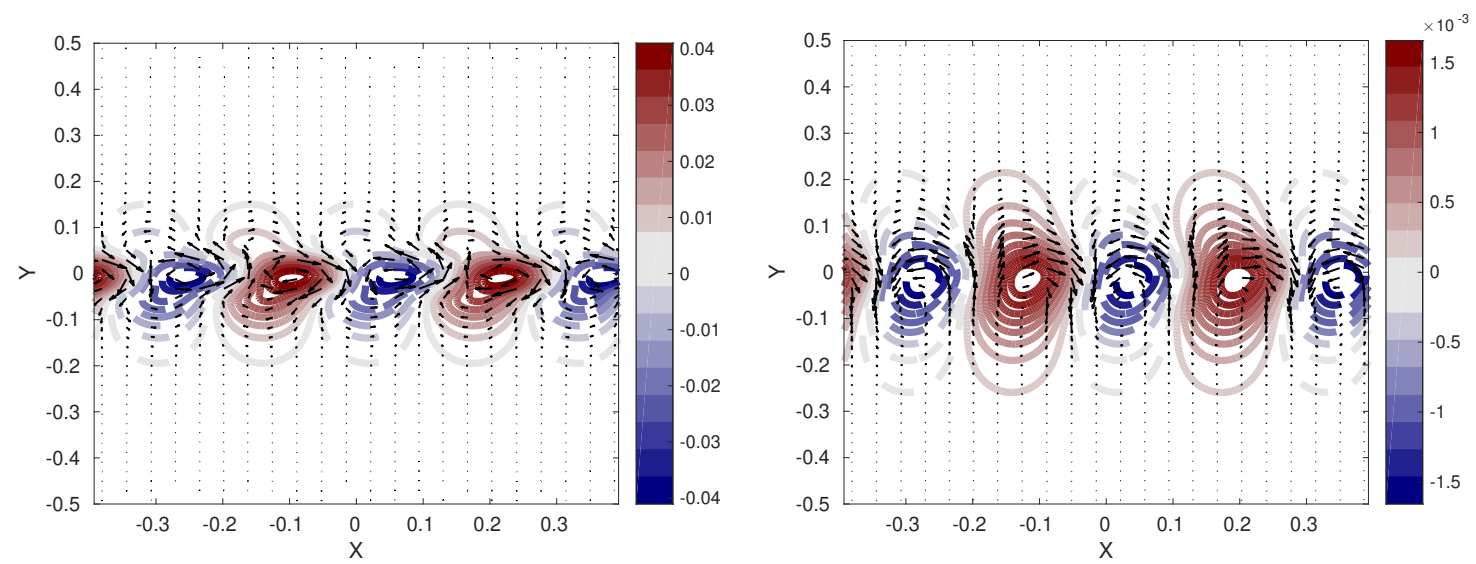

Figure A2. Geostrophic stream-functions of the most unstable mode on the $f$-plane in the upper layer (left panel) $\left[\psi_{2}=\eta_{1}+s \eta_{2}\right]$ and the lower layer (right panel) $\left[\psi_{1}=\eta_{1}+\eta_{2}\right]$. Positive (negative) values are represented by solid (dashed) lines, and the velocity field is represented by arrows. $k=20, R o=0.15, U_{0}=0.045, a=20, H_{01}=1 / 3, H_{02}=$ $2 / 3, \Phi=13^{\circ} \mathrm{N}, \bar{\beta}=0$ (Colour online).

\section{Appendix A: Stability of the easterly jet on the $f$-plane}

In this Appendix we present the results of the auxiliary investigation of the stability of the easterly upper-layer jet on the $f$-plane. The stability diagram, and the phase-portrait and meridional cross-section of the most unstable mode are displayed, respectively in Figures A1, A2, and A3. We present in Figure A1 the results both for large and small Burger numbers of the jet. The former case is close to that of Lambaerts et al. (2012), with the Rossby number being close, too, and the results quantitatively and qualitatively similar, which is, thus, a successful benchmark. The latter case highlights the dependence of the results on $B u$, which was not analyzed in Lambaerts et al. (2012). 

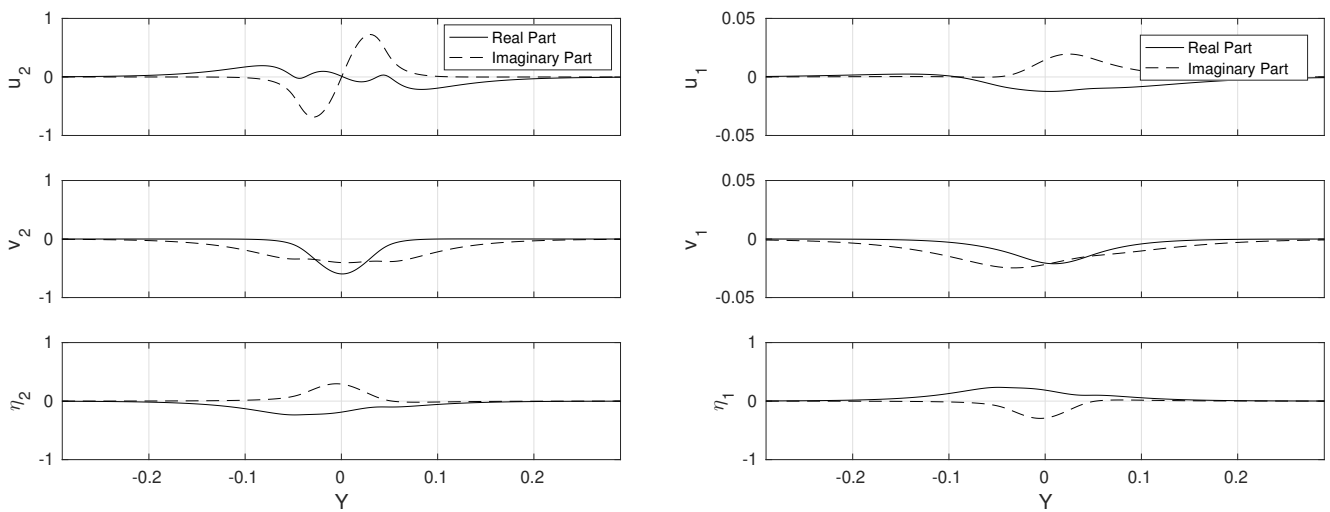

Figure A3. Meridional cross-sections of $u_{i}, v_{i}, \eta_{i}$ of the most unstable mode on the $f$-plane shown in the Figure A2, in the upper layer (left panel) and the lower layer (right panel) (Colour online).

\section{References}

Bouchut, F., Lambaerts, J., Lapeyre, G. and Zeitlin, V., Fronts and nonlinear waves in a simplified shallowwater model of the atmosphere with moisture and convection. Phys. Fluids, 2009, 21, 116604.

Bouchut, F. and Zeitlin, V., A robust well-balanced scheme for multi-layer shallow water equations. Disc. Cont. Dyn. Syst., 2010, B13, 739-758.

Bracken, W. and Bosart, L., The role of synoptic-scale flow during tropical cyclogenesis over the North Atlantic Ocean. Mon. Weather Rev., 2000, 128, 353-376.

Brammer, A. and Thorncroft, C., Variability and evolution of African easterly wave structures and their relationship with tropical cyclogenesis over the Eastern Atlantic. Mon. Weather Rev., 2015, 143, 4975-4995.

Burpee, R., The origin and structure of easterly waves in the lower troposphere of North Africa. J. Atmos. Sci., 1972, 29, 1165-1184.

Chen, T., Wang, S. and Clark, A., North Atlantic hurricanes contributed by African easterly waves North and South of the African easterly jet. J. Climate, 2008, 21, 6767-6776.

Cook, K., Generation of the African easterly jet and its role in determining west African precipitation. $J$. Climate, 1999, 12, 77-90.

Cornforth, R., Mumba, Z., Parker, D.J., Berry, G., Chapelon, N., Diakaria, K., Diop-Kane, M., Ermert, V., Fink, A.H., Knippertz, P., Lafore, J.P., Laing, A., Lepape, S., Maidment, R., Methven, J., Orji, B., Osika, D., Poan, E., Roca, R., Rowell, S., Smith, R., Spengler, T., Taylor, C.M., Thorncroft, C., Vincendon, J.C., Yorke, C. and Thorncroft, C., Ch.2 Synoptic systems. In Meteorology of Tropical West Africa, pp. 40-89, 2017 (John Wiley and Sons: Hoboken NJ).

Diaz, M. and Aiyyer, A., Absolute and convective instability of the African easterly jet. J. Atmos. Sci., 2015, 72, 1805-1826.

Dieng, A., Sall, S., Eymard, L., Leduc-Lebailleur, M. and Lazar, A., Trains of African easterly waves and their relationship to tropical cyclone genesis in the Eastern Atlantic. Mon. Weather Rev., 2017, 145, 599-616.

Hall, N., Kiladis, G. and Thorncroft, C., Three-dimensional structure and dynamics of African easterly waves. Part II: Dynamical Modes. J. Atmos. Sci., 2006, 63, 2231-2245.

Hsie, J. and Cook, K., Generation of African easterly wave disturbances: relationship to the African easterly jet. Mon. Weather Rev., 2005, 133, 1311-1327.

Hsie, J. and Cook, K., On the instability of the African easterly jet and the generation of African waves: reversals of the potential vorticity gradient. J. Atmos. Sci., 2008, 65, 2130-2151. 
Katsaros, K., Evaporation And Humidity. In Encyclopedia of Ocean Sciences, edited by J.H. Steele, pp. 870 877, 2001 (Academic Press: Oxford).

Kuo, H., A two-layer model study of the combined barotropic and baroclinic instability in tropics. J. Atmos. Sci., 1978, 35, 1840-1860.

Lafore, J.P., Beucher, F., Peyrille, P., Diongue-Niang, A., Chapelon, N., Bouniol, D., Caniaux, G., Favot, F., Ferry, F., Guichard, F., Poan, E., Roehrig, R. and Vischel, T., A multi-scale analysis of the extreme rain event of Ouagadougou in 2009. Q. J. Roy. Met. Soc., 2017, 143, 3094-31109.

Lahaye, N. and Zeitlin, V., Understanding instabilities of tropical cyclones and their evolution with a moist convective rotating Shallow-Water model. J. Atmos. Sci., 2016, 73, 505-523.

Lambaerts, J., Lapeyre, G. and Zeitlin, V., Moist versus dry barotropic instability in a Shallow-Water model of the atmosphere with moist convection. J. Atmos. Sci., 2011a, 68, 1234-1252.

Lambaerts, J., Lapeyre, G. and Zeitlin, V., Moist versus dry baroclinic instability in a simplified two-Layer atmospheric model with condensation and latent heat release. J. Atmos. Sci., 2012, 69, 1405-1426.

Lambaerts, J., Lapeyre, G., Zeitlin, V. and Bouchut, F., Simplified two-layer models of precipitating atmosphere and their properties. Phys. Fluids, 2011b, 23, 046603.

Liu, Y., Kurganov, A. and Zeitlin, V., Moist-convective thermal rotating shallow water model. Phys. Fluids, 2020, 32, 066601.

Maslowe, S., Barotropic instability of the Bickley jet. J. Fluid Mech., 1991, 229, 417-426.

Parker, D., A simple model of coupled synoptic waves in the land surface and atmosphere of the northern Sahel. Q. J. Roy. Met. Soc., 2008, 134, 2173-2184.

Pytharoulis, I. and Thorncroft, C., The low-level structure of African easterly waves in 1995. Mon. Weather Rev., 1999, 127, 2266-2280.

Rostami, M. and Zeitlin, V., Influence of condensation and latent heat release upon barotropic and baroclinic instabilities of atmospheric vortices in a rotating shallow water model on the f-plane. Geoph. Astrophys. Fluid Dyn., 2017, 111, 1-31.

Rostami, M. and Zeitlin, V., Improved moist-convective rotating shallow water model and its application to instabilities of hurricane-like vortices. Q. J. Roy. Met. Soc., 2018, 144, 1450-1462.

Rostami, M. and Zeitlin, V., Evolution, propagation and interactions with topography of hurricane-like vortices in a moist-convective rotating shallow-water model. J. Fluid. Mech., 2020, 902, A24.

Rostami, M., Zeitlin, V. and Montabone, L., On the role of spatially inhomogeneous diabatic effects upon the evolution of Mars' annular polar vortex. Icarus, 2018, 314, 376 - 388.

Rostami, M., Zeitlin, V. and Spiga, A., On the dynamical nature of Saturn's North Polar hexagon. Icarus, 2017, 297, $59-70$.

Schecter, D. and Dunkerton, T., Hurricane formation in diabatic Ekman turbulence. Q. J. Roy. Met. Soc., 2009, 135, 823-840.

Simmons, A., A note on the instability of the African easterly jet. J. Atmos. Sci., 1977, 34, 1670-1674.

Swaters, G., On the evolution of near-singular modes of the Bickley jet. Phys. Fluids, 1999, 11, 2546.

Thorncroft, C. and Hoskins, B., An idealized study of African easterly waves. I: A linear view. Q. J. Roy. Met. Soc., 1994a, 120, 953-982.

Thorncroft, C. and Hoskins, B., An idealized study of African easterly waves. II: A nonlinear view. Q. J. Roy. Met. Soc., 1994b, 120, 983-1015.

Wu, M., Reale, O., Schubert, S., Suarez, M. and Thorncroft, C., African easterly jet: barotropic instability, waves, and cyclogenesis. J. Climate, 2012, 25, 1489-1510.

Zeitlin, V., Geophysical Fluid Dynamics: Understanding (almost) Everything with Rotating Shallow Water 
Models, 2018 (Oxford University Press, Oxford). 